\title{
Non-equilibrium quantum noise in chiral Luttinger liquids
}

\author{
C. de C. Chamon \\ Department of Physics, Massachusetts Institute of Technology, Cambridge, MA 02139
}

D. E. Freed

The Mary Ingraham Bunting Institute, Radcliffe Research and Study Center, Harvard University, Cambridge, MA 02138

X. G. Wen

Department of Physics, Massachusetts Institute of Technology, Cambridge, MA 02139

\begin{abstract}
We study non-equilibrium noise in Chiral Luttinger Liquids using the Landauer-Buttiker Scattering approach, obtaining the current/voltage noise spectrum for a four-terminal measurement scheme. Experimental consequences of the tunneling of charges are present in the four-terminal measurement of both the low-frequency shot noise ( $\omega$ near 0$)$, and the high-frequency Josephson noise $(\omega$ near $\left.\omega_{J}=e^{*} V / \hbar\right)$. Within perturbation theory, an algebraic singularity is present (to all orders) at the Josephson frequency $\omega_{J}=e^{*} V / \hbar$, whose position depends on the charge $e^{*}$ of the tunneling particles, either electrons or fractionally charged quasiparticles. We show in a non-perturbative calculation for an exactly solvable point that the singularity at the quasiparticle frequency exists only in the limit of vanishing coupling, whereas the singularity at the electron frequency is present for all coupling strengths. The vanishing coupling limit corresponds to perfectly quantized Hall conductance in the case of quasiparticle tunneling between edge states in the fractional quantum Hall regime, and thus tunneling destroys the singularity at the quasiparticle frequency concomitantly with the quantized current.
\end{abstract}

PACS: 72.10.Bg, 73.20.Dx, 73.40.Gk, 73.50.Fq, 73.50.Td

\section{INTRODUCTION}

Recently it was realized that a strongly correlated $1 \mathrm{D}$ system, namely a Chiral Luttinger liquid ( $\chi \mathrm{LL}$ ), exists in the edges of fractional quantum Hall (FQH) liquids [1]. Because of their chiral nature, i.e., the excitations in a given branch move only in one direction, spatially separated branches cannot interfere and cause localization of states. In contrast, non-chiral Luttinger liquids are extremely sensitive to the presence of even the smallest amounts of impurity in the sample, for in 1D all states are localized, and long enough wires will behave as insulators. The characteristic property of (chiral) Luttinger liquids is that the tunneling conductance between the edge states has a power law dependence on the temperature $\sigma \propto T^{2(g-1)}$, where $g$ depends on the filling factor $\nu$ of the FQH state, taking the values $g=\nu$ or $g=\nu^{-1}$ depending on the tunneling geometry [2 5]. By experimentally studying the tunneling between edge states in the FQH regime using a point contact geometry, Milliken, Umbach, and Webb [6] found this type of power law dependence of the tunneling conductance on the temperature. Their finding is consistent with the theoretical prediction $\sigma \propto T^{4}$ for the $\nu=1 / 3 \mathrm{FQH}$ state [2,7].

The experimental confirmation of the Luttinger liquid behavior in tunneling between edge states has boosted theoretical interest in further studies of properties of the conductance [8 10]. An exact solution for the conductance has been obtained using the thermodynamic Bethe Ansatz, and an exact duality between the $g$ and $1 / g$ cases has been shown [11,12] in the context of the tunneling current, as suggested in Ref. [13]. The rich behavior of tunneling in chiral Luttinger liquids extends well beyond transport measurements alone 14 16. One should expect, based on experience in non-interacting systems, that the noise spectrum contains information not attainable, in the most general case, from just transport measurements. In general, the shape of the noise spectrum is determined by the dynamical properties of the system, which in turn contain information about the excited states. Even for non-interacting electronic systems, non-trivial structures appear in the noise [17 19], the simplest example being the suppression of classical shot noise due to quantum statistics. In chiral Luttinger liquids, the tunneling particles sometimes carry fractional charge and fractional statistics, and thus such strongly correlated 1D systems also provide the natural experimental realization for the study of features that arise in the noise spectrum for generalized quantum statistics.

In reference [15], the noise spectrum of the tunneling current between edge states directly at the point contact was calculated perturbatively. To low orders in the tunneling amplitude, we found that there was a singularity at $\omega=0$; for small $\omega$ the noise spectrum has the form $S_{S N}+S_{\operatorname{sing}}(\omega)$, where $S_{S N}$ is the zero-frequency shot noise and $S_{\text {sing }}(\omega)=c|\omega|$. The slope $c$ of the $|\omega|$ singularity has a strong non-linear dependence on the applied voltage $V$ $\left(c \propto(2 g-1)^{2} V^{4(g-1)}\right)$, which is another signature of Luttinger liquid behavior (to be contrasted with the case of 
non-interacting electrons, $g=1$, where the slope is independent of $V$ ). The exponent $g$ characterizes the Luttinger liquid behavior. This low-frequency part of the spectrum is the one more easily accessible experimentally. Secondly, there is another singularity at $\omega=\omega_{J}$ where $\omega_{J}=e^{*} V / \hbar$ is the Josephson frequency of the electron $\left(e^{*}=e\right)$ or quasiparticle $\left(e^{*}=\nu e\right)$ that tunnels through the point contact. The shape of this singularity depends on $g$ and goes as $\left|\omega \pm \omega_{J}\right|^{2 g-1}$. Measurements of the location of this singularity would give the value $e^{*}$ of the charge of the carriers of the current, which would be yet another way of observing fractional charge from noise measurements. The method originally suggested is to measure the shot noise, which for small tunneling amplitude is related to the tunneling current $I_{t}$ by $S_{S N}=2 e^{*} I_{t}$ 14 16]. Lastly, for $g>1$, we found that the singularities at both $\omega=0$ and $\omega=\omega_{J}$ should persist to all orders in perturbation theory.

These results present a puzzle which we describe below and address in this paper. The case of $g=\nu<1$ corresponds to a single quantum Hall droplet with a constriction. In this case, quasiparticles can tunnel across the constriction, from one edge to the other (see Fig. 1a). These quasiparticles have fractional charge $e^{*}$, given by $\nu e$. If the constriction is made narrower, the tunneling amplitude will increase. As the constriction is further narrowed, eventually the droplet will break into two disconnected pieces, and now only electrons will be able to tunnel from one edge to the other (see Fig. 1b). Their tunneling should once again behave like tunneling in a chiral Luttinger liquid, but with new exponent $\tilde{g}=1 / g$ and charge equal to $e$. This is the physical picture behind the duality seen in reference [12]; as the tunneling amplitude is increased (or the voltage is decreased) $g$ goes to $1 / g$. Similarly, if we start with the two quantum Hall droplets with exponent $\tilde{g}$ and increase the tunneling amplitude of the electron, eventually we will obtain the single droplet picture with exponent $g=1 / \tilde{g}$.

In light of this duality and the results of reference [15], the following question arises. If we start with the two disconnected droplets then we expect the singularity in the noise to occur at multiples of $\tilde{\omega}_{J}=e V / \hbar$, the Josephson frequency for the electron. As the tunneling amplitude is increased, at some point we expect the singularity at the Josephson frequency for the quasiparticle, $\omega_{J}=e^{*} V / \hbar$, to appear. However, according to the perturbative calculations, to all orders in the electron tunneling amplitude the quasiparticle singularity does not appear. In this paper we will address the question of what happens to this quasiparticle singularity and show how these two seemingly contradictory statements are resolved in the special case of $g=1 / 2$ and $\tilde{g}=2$. In short, our results suggest that the singularity at the quasiparticle Josephson frequency $\omega_{J}=e^{*} V / \hbar$ is destroyed by non-perturbtive effects. In some sense, the singularity obtained by perturbative calculations is smeared for finite tunneling strength. This question is of interest because the location of the singularities tells us which particles are tunneling and, as mentioned above, should give a way to measure the fractional charge of the quasiparticles. The results in this paper suggest what may be happening at other values of $g$ also, and we are currently working on this question.

One of the tools we use in this paper is the Landauer-Buttiker scattering approach. The geometry is illustrated in Figure 2. The choice of the Landauer-Buttiker approach is justified for a number of reasons. The chiral nature of the system under study naturally poses the problem in terms of incoming and outgoing scattering states to and from the point contact region. The incoming branches should be in equilibrium with their respective reservoirs of departure, and should be insensitive to the tunneling of charges in the tunneling region shown in Fig. 2. This is so because information on tunneling events cannot propagate in the direction opposite to the incoming branch chirality. Also, the Landauer-Buttiker approach and the chiral nature of the system suggest naturally a four-terminal geometry for experimental measurements, probing voltage fluctuations in the two incoming and two outgoing branchs. The tunneling takes place in the point contact, or scattering region, which is not directly accessible by the probing leads. Auto-correlations of current/voltage fluctuations measured in the four terminals, as well as cross-correlations between different terminals, are the experimental probes that should allow the remote measurement of the tunneling events and noise spectrum.

The paper is organized as follows. In section II we briefly review the bosonization scheme for chiral Luttinger liquids. In section III we obtain the noise spectrum perturbatively for the four terminal geometry, using the Keldysh nonequilibrium formalism. We show that only the noise spectrum for the outgoing branches is affected by the tunneling, whereas the incoming branches are completely insensitive to the charge transfer between the edges. This is consistent with the Landauer-Buttiker picture and the chirality of the system. The noise spectrum for the incoming branches can thus serve as reference level for the measurement of the excess noise on the outgoing branches due to tunneling. The noise spectrum obtained contains interesting structures both at low and high frequencies. The tunneling excess noise vanishes for frequencies above the Josephson frequency $\omega_{J}=e^{*} V / \hbar$. The issue of how the singularity moves from the quasiparticle frequency to the electron frequency is resolved in section IV, where we use the Landauer-Buttiker approach to solve exactly for the noise spectrum in the case of $g=1 / 2$, for which the problem can be cast as a free fermion problem. We show that the singularity at the quasiparticle frequency is smeared for finite tunneling and is not a true singularity, whereas the singularity at the electron frequency survives for all non-zero coupling. In section $\mathrm{V}$ we discuss the duality when $g=1 / 2$ goes to $\tilde{g}=2$, which we show is not exact in the naive sense for the case of noise, in contrast to the case of conductance. We find that the noise spectrum of the current correlations on a single branch (auto-correlations) satisfies the duality relation, while current correlations between distinct branches 
(cross-correlations) do not satisfy the naive duality relation. We show that the correct dual Lagrangian to the $g=1 / 2$ theory is the $g=2$ theory plus a neutral density-density coupling, which has the same dimension as the tunneling operator. The effect of the neutral coupling appears in the noise, but not in the conductance.

\section{EDGE STATE TUNNELING}

In this section we shall briefly review the bosonization scheme for edge states in the FQH effect (for a thorough review, see Ref. [20]). The Lagrangian we will use is better cast in this bosonic language.

The right and left moving excitations along the edges can be described by boson fields $\phi_{R, L}$. Right and left moving electron and quasiparticle operators on the edges of a FQH liquid can be written as $\Psi_{R, L}(t, x) \propto e^{ \pm i \sqrt{g} \phi_{R, L}(t, x)}$, where $g$ is related to the FQH bulk state. For example, for a Laughlin state with filling fraction $\nu=1 / m$ we have $g=m$ for electrons and $g=1 / m$ for quasiparticles carrying fractional charge $e / m$. The $\phi_{R, L}$ fields satisfy the equal-time commutation relations

$$
\left[\phi_{R, L}(t, x), \phi_{R, L}(t, y)\right]= \pm i \pi \operatorname{sign}(x-y) .
$$

The dynamics of $\phi_{R, L}$ is described by

$$
\mathcal{L}_{R, L}=\frac{1}{4 \pi} \partial_{x} \phi_{R, L}\left( \pm \partial_{t}-v \partial_{x}\right) \phi_{R, L}
$$

where $v$ is the velocity of edge excitations (which we will set to 1 ). Density operators can be defined in terms of the $\phi_{R, L}$ through $\rho_{R, L}=\frac{\sqrt{\nu}}{2 \pi} \partial_{x} \phi_{R, L}$. Here, for convenience, we have set the unit charge in the definition of the density to be the electron charge $e$, so that $e=1$ and $e^{*}=\nu$. One can verify that $\left[\rho_{R, L}(t, x), \Psi_{R, L}^{\dagger}(t, y)\right]=\sqrt{\nu g} \Psi_{R, L}^{\dagger}(t, y) \delta(x-y)$, so that indeed the cases $g=\nu^{-1}$ and $g=\nu$ correspond to electron and quasiparticle charged operators, respectively.

The tunneling operators from right to left moving branches and vice-versa can be written as $\Psi_{L}^{\dagger} \Psi_{R}$ and $\Psi_{R}^{\dagger} \Psi_{L}$. Thus we can write, in terms of $\phi=\phi_{R}+\phi_{L}$, the following total Lagrangian density:

$$
\mathcal{L}=\frac{1}{8 \pi}\left[\left(\partial_{t} \phi\right)^{2}-v^{2}\left(\partial_{x} \phi\right)^{2}\right]-\Gamma \delta(x) e^{i \sqrt{g} \phi(t, 0)}+\text { H.c. },
$$

with $\phi$ satisfying $\left[\phi(t, x), \partial_{t} \phi(t, y)\right]=4 \pi i \delta(x-y)$. In the following we will set the edge velocity $v=1$.

A voltage difference between the two edges of the $\mathrm{QH}$ liquid can be easily introduced in the model by letting $\Gamma \rightarrow \Gamma e^{-i \omega_{0} t}$, where $\omega_{0} \equiv \omega_{J} \equiv e^{*} V / \hbar$, with $e^{*}=e$ for electron tunneling and $e^{*}=e / m$ for quasiparticle tunneling.

In the following sections we will study non-equilibrium noise in chiral Luttinger liquids described by the model above.

\section{PERTURBATIVE APPROACH}

In this section we treat the tunneling between edge states perturbatively, and obtain the noise spectrum for the current/voltage fluctuations at the four leads as shown in Fig. 2. In the figure we separate the branches into their right and left moving components, as well as incident and scattered ones. Right and left branches are incoming or outgoing depending on their position relative to the scatterer:

$$
\begin{array}{cccc}
\text { Incident } & \phi_{R}(t, x<0) & \text { and } & \phi_{L}(t, x>0) \\
\text { Scattered } & \phi_{R}(t, x>0) & \text { and } & \phi_{L}(t, x<0) .
\end{array}
$$

Both the currents and the densities at the four terminals can be related to the fields $\phi_{R, L}$. The densities are simply given by $\rho_{R, L}=\frac{\sqrt{\nu}}{2 \pi} \partial_{x} \phi_{R, L}$. Voltage measurements probe these densities. The currents at the four terminals can be trivially related to the densities at those same terminals through the continuity equation for $x \neq 0$. The currents are given by $j_{R, L}= \pm \frac{\sqrt{\nu}}{2 \pi} \partial_{x} \phi_{R, L}$, with positive currents flowing to the right. By choosing the convention that positive currents flow in the direction of the arrows in Fig. 2 , we can write new currents $\tilde{j}_{R, L}= \pm j_{R, L}=\rho_{R, L}$. It then becomes transparent that there is a tight relationship between current and voltage in the chiral branches. For example, measuring the noise in either the current or the voltage yields information about the other. This kind of relationship between voltage and current noise was obtained in Ref. [14]. We will thus focus on the calculation of density-density correlations, for these will give us information on both current and voltage noise. 
We will label the densities at the four terminals shown in Fig. 2 by $\rho_{i}, i=1,2,3,4$. In terms of the right and left moving fields we have:

$$
\begin{array}{ll}
\rho_{1}(t)=\rho_{R}\left(t, x_{1}\right) & \rho_{3}(t)=\rho_{R}\left(t, x_{3}\right) \\
\rho_{2}(t)=\rho_{L}\left(t, x_{2}\right) & \rho_{4}(t)=\rho_{L}\left(t, x_{4}\right),
\end{array}
$$

where $x_{1}, x_{4}<0, x_{2}, x_{3}>0$. The noise spectrum of the density fluctuations in terminals $i, j$ is obtained from the correlations between the densities $\rho_{i}, \rho_{j}$ :

$$
S_{i j}(\omega)=S_{j i}(-\omega)=\int_{-\infty}^{\infty} d t e^{i \omega t}\left\langle\left\{\rho_{i}(t), \rho_{j}(0)\right\}\right\rangle
$$

These quantities are calculated perturbatively in Appendix A, using the techniques in Ref. [15. The components with $i \neq j$ are very sensitive to phases which depend on the position of the probes $x_{i}$ and $x_{j}$. These phases cancel in the case of auto-correlations, i.e., when $i=j$. The quantities $S_{i i}(\omega)$, which correspond to the noise spectrum obtained entirely from one of the four probes for $i=1$ to 4 , are thus the most robust measurements of fluctuations, because when they are extracted away from the junction they are independent of the position $x_{i}$ where they are taken.

To second order in perturbation theory, $S_{i i}$ is given by:

$$
\begin{aligned}
& S_{11}(\omega)=S_{22}(\omega)=S^{(0)}(\omega) \\
& S_{33}(\omega)=S_{44}(\omega)=S^{(0)}(\omega)+S^{(2)}(\omega),
\end{aligned}
$$

where

$$
\begin{aligned}
S^{(0)}(\omega) & =\frac{\nu}{2 \pi}|\omega|, \\
S^{(2)}(\omega) & =\frac{4 \pi \nu g}{\Gamma(2 g)}|\Gamma|^{2}|| \omega|-| \omega_{J}||^{2 g-1} \theta\left(\left|\omega_{J}\right|-|\omega|\right) .
\end{aligned}
$$

Using the perturbative result to order $|\Gamma|^{2}$ for the tunneling current $I_{t}=\frac{2 \pi}{\Gamma(2 g)} e^{*}|\Gamma|^{2} \omega_{J}^{2 g-1}$ [2], $S^{(2)}(\omega)$ can be wrtitten as

$$
S^{(2)}(\omega)=\left.2 e^{*} I_{t}|1-| \frac{\omega}{\omega_{J}}\right|^{2 g-1} \theta\left(\left|\omega_{J}\right|-|\omega|\right) .
$$

Notice that the effects of tunneling are contained in $S^{(2)}(\omega)$, and only appear in the outgoing branches, terminals $i=3,4$. The incoming branches are insensitive to the tunneling between edges, due to the chiral nature of the system. Information about what goes on in the junction cannot propagate in the direction opposite to the chirality of the branch, and therefore the noise in the incoming branches is independent of the tunneling of charged particles between edges. This result of chirality is clear within the Landauer-Buttiker scattering approach. Another physical consequence closely related to this is the fact that the average voltage along the branches remains constant outside the scattering region.

The second point to notice from Eq. (11) is that to order $|\Gamma|^{2}$ the noise in the outgoing branches that is in excess to the noise in the incoming branches has a singularity at the Josephson frequency $\omega_{J}$, vanishing for $\omega>\omega_{J}$, as illustrated in Fig. 3. The non-equilibrium voltage $V$ determines the frequency scale $\omega_{J}=e^{*} V / \hbar$, up to which there is structure in the excess noise due to tunneling. Such vanishing of the excess noise spectrum past a frequency set by the non-equilibrium voltage should be familiar to readers accustomed to noise in non-interacting systems $(g=1)$, in which the excess noise goes to zero linearly at the Josephson frequency [21]. This point will be illustrated further in the next section, when we will have at hand the exact solution for the noise spectrum in the case of $g=1 / 2$. The strong coupling limit of the solution for $g=1 / 2$ also gives us the solution for $g=2$, which we shall use for comparison purposes.

The last, and most important, point about this high frequency singularity in the noise spectrum is in regard to the connection between the two dual pictures illustrated in Fig. 1. In Ref. [15] it was pointed out that the singularity at the Josephson frequency remained to all orders in perturbation theory. However, the perturbative expansion for the geometries in Figs. 1a and 1b yields two distinct frequencies, namely the quasiparticle frequency $\omega_{q p}=\nu e V / \hbar$ when quasiparticles are the tunneling charges (Fig. 1a), and the electron frequency $\omega_{e l}=e V / \hbar$, when electrons are the tunneling current carriers (Fig. 1b). These configurations are connected in the sense that one is the strong coupling limit of the other, and thus there should be a non-perturbative mechanism by which the singularity moves from one place to the other. This was the clearest open question in Ref. [15], and which we can answer by focusing on the 
exactly solvable case of $g=1 / 2$. Another exactly solvable point is the trivial case $g=1$, which unfortunately cannot be used to address this issue of the singularity in the noise spectrum because in this case the two frequencies $\omega_{e l}$ and $\omega_{q p}$ coincide.

Before answering the question about the high frequency singularity, we will close this section with the implications of tunneling between edge states to the low frequency noise measured in the four terminal geometry. In Ref. [15], a correction to the low frequency shot noise spectrum was found, which corresponded to an $|\omega|$ singularity, or a cusp, in the noise spectrum. This correction was found to order $|\Gamma|^{4}$, while to order $|\Gamma|^{2}$ the low frequency corrections to the flat shot noise started as $\propto \omega^{2}$. In the four terminal geometry proposed in this paper, what is probed is not the tunneling current in the junction area (as in Ref. [15]), but its consequences in the current/voltage in the four terminals away from the scattering region. The four terminal measurement, as seen from Eq. (11), does have a correction $\propto|\omega|$ to order $|\Gamma|^{2}$. For $\omega \ll \omega_{J}$ we have, for example,

$$
\begin{aligned}
S_{33}(\omega)-S_{33}^{V=0}(\omega) & =S^{(2)}(\omega)=S_{33}(\omega)-S_{11}(\omega) \\
& =2 e^{*} I_{t}|1-| \frac{\omega}{\omega_{J}}||^{2 g-1} \theta\left(\left|\omega_{J}\right|-|\omega|\right) \\
& \approx 2 e^{*} I_{t}\left[1-(2 g-1)\left|\frac{\omega}{\omega_{J}}\right|\right] .
\end{aligned}
$$

One recovers the classical shot noise expression for $\omega=0$. Notice that, since these results are valid only to order $|\Gamma|^{2}$, there is no correction to the classical shot noise expression for $\omega=0$. Corrections appear at order $|\Gamma|^{4}$ (see Ref. $[15 \mid$ ). Also notice that the non-zero $\omega$ corrections to the shot noise depend on whether $g$ is larger or smaller then $1 / 2$. For $g>1 / 2$, the difference between the outgoing and incoming spectra (the $S_{33}(\omega)-S_{11}(\omega)$ above, for example) decreases with $\omega$, whereas for $g<1 / 2$ it increases.

\section{SCATTERING APPROACH FOR $G=1 / 2$}

In this section we will use the Landauer-Buttiker Scattering approach to obtain an exact solution for the noise when $g=1 / 2$. In this approach, we use the quantum equations of motion derived from the Hamiltonian to solve for the scattering states. These scattering states describe free left movers and right movers that are incident on the impurity and then are reflected or scattered by the impurity. The solutions for these states can be used to calculate the conductance and the noise in the various branches.

The advantage of focussing on $g=1 / 2$ is that for this value of $g$ the system can be described by free fermions 22,23, making it straightforward to solve for the scattering states. However, already at $g=1 / 2$, we expect to see singularities in the noise at $\omega_{J}=e^{*} V / \hbar$, corresponding to quasiparticle tunneling. As the tunneling amplitude $\Gamma$ increases (or $V$ decreases), we expect to obtain the dual picture at $g=2$, with electrons tunneling and a singularity at $\mathrm{eV} / \hbar$. Thus the full solution at $g=1 / 2$ will show us what happens to the quasiparticle singularity as $\Gamma$ is increased. The hope is that the qualitative behavior of these results will also apply for other values of $g$.

When $g=1 / 2$, the Hamiltonian for the system is given by

$$
H=H_{R}^{0}+H_{L}^{0}+\Gamma e^{-i \omega_{0} t} e^{\frac{i}{\sqrt{2}}\left(\phi_{R}(t, 0)+\phi_{L}(t, 0)\right)}+\Gamma^{*} e^{i \omega_{0} t} e^{-\frac{i}{\sqrt{2}}\left(\phi_{R}(t, 0)+\phi_{L}(t, 0)\right)}
$$

where $H_{R, L}^{0}$ are the free Hamiltonians for the right and left moving fields, and $\omega_{0}=e^{*} V / \hbar$, with $e^{*}=e / 2$.

The Hamiltonian can be recast in terms of new chiral fields $\phi_{\mp}(t, x)=\frac{1}{\sqrt{2}}\left(\phi_{R}(t, x) \pm \phi_{L}(t,-x)\right)$ :

$$
H=H_{+}^{0}+H_{-}^{0}+\Gamma e^{-i \omega_{0} t} e^{i \phi_{-}(t, 0)}+\Gamma^{*} e^{i \omega_{0} t} e^{-i \phi_{-}(t, 0)} .
$$

The densities of the new fields $\rho_{ \pm}=\frac{1}{2 \pi} \partial_{x} \phi_{ \pm}$are related to the densities $\rho_{R, L}=\frac{\sqrt{1 / 2}}{2 \pi} \partial_{x} \phi_{R, L}$ by $\rho_{ \pm}(t, x)=\rho_{R}(t, x) \pm$ $\rho_{L}(t,-x)$. Notice that the $\phi_{ \pm}$fields are decoupled in Eq. 14 , and the Hamiltonian for $\phi_{+}$is simply the free $H_{+}^{0}$. The Hamiltonian for $\phi_{-}$can be fermionized by defining $\eta(t, x) \equiv \frac{1}{\sqrt{2 \pi}}: e^{i \phi_{-}(t, x)}:$. One can check that $\eta$ defined as such satisfies the proper commutation relations $\left\{\eta(t, x), \eta^{\dagger}(t, y)\right\}=\delta(x-y)$ [24].

In terms of the fermionic fields $\eta, \eta^{\dagger}$, the Hamiltonian $H_{-}$is:

$$
H_{-}=\int d x\left\{\eta^{\dagger}(x)\left[-i \frac{\partial}{\partial x}-\omega_{0}\right] \eta(x)+\sqrt{2 \pi} \delta(x)\left[\Gamma \eta(x)+\Gamma^{*} \eta^{\dagger}(x)\right]\right\},
$$


where we absorbed the oscillating phases $e^{i \omega_{0} t}$ into a redefinition of the chemical potential. The Hamiltonian above contains terms linear in the fermionic fields $\eta$ and $\eta^{\dagger}$, which prevent a direct calculation of the commutators that would give us the equations of motion for the fields. This problem can be circumvented by redefining the fermionic fields to be $\psi(t, x)=\eta(t, x) f$, with $f=C+C^{\dagger}$ and $\left\{C, C^{\dagger}\right\}=1$, as in Ref. 23]. More formally, such a transformation can be constructed from the proper handling of the zero modes of the bosonic fields $\phi$ [1,25], and one can identify $f$ with $(-1)^{F}$, the fermion counting operator commonly used to switch from periodic to anti-periodic boundary conditions in fermionic conformal field theories.

The Hamiltonian we will use in the exact solution of the noise spectrum for the $g=1 / 2$ case is the one written in terms of the $\psi, \psi^{\dagger}$ fields and $f$ :

$$
H_{-}=\int d x\left\{\psi^{\dagger}(x)\left[-i \frac{\partial}{\partial x}-\omega_{0}\right] \psi(x)+\sqrt{2 \pi} \delta(x)\left[\Gamma \psi(x) f+\Gamma^{*} f \psi^{\dagger}(x)\right]\right\}
$$

where the non-vanishing equal-time commutation relations between $\psi(x), \psi^{\dagger}(x)$ and $f$ are

$$
\left\{\psi(x), \psi^{\dagger}\left(x^{\prime}\right)\right\}=\delta\left(x-x^{\prime}\right),\{\psi(x), f\}=0,\{f, f\}=2 .
$$

The density $\rho_{-}$can be written in terms of the fields $\psi$ and $\psi^{\dagger}$ as $\rho_{-}(x)=\psi^{\dagger}(x) \psi(x)$, so that all correlations between $\rho_{-}$'s can be derived from the correlations of the fermions. The fermionic model is solved using the equations of motion obtained by commuting the operators $\psi(x)$ and $f$ with the Hamiltonian:

$$
\begin{aligned}
-i \partial_{t} \psi(x) & =[H, \psi(x)]=\left(i \partial_{x}+\omega_{0}\right) \psi(x)+\sqrt{2 \pi} \Gamma^{*} f \delta(x), \\
-i \partial_{t} \psi^{\dagger}(x) & =\left[H, \psi^{\dagger}(x)\right]=\left(i \partial_{x}-\omega_{0}\right) \psi^{\dagger}(x)-\sqrt{2 \pi} \Gamma f \delta(x),
\end{aligned}
$$

and

$$
-i \partial_{t} f=[H, f]=2 \sqrt{2 \pi}\left[\Gamma \psi(0)-\Gamma^{*} \psi^{\dagger}(0)\right] .
$$

According to these equations, for $x \neq 0$, the field $\psi$ satisfies the free equation of motion for a rightmover with energy shifted by $\omega_{0}$ :

$$
\left(i \partial_{x}+i \partial_{t}+\omega_{0}\right) \psi=0
$$

At $x=0$, it picks up a discontinuity because of the impurity. In order to preserve unitarity and obtain the proper commutation relations in the solutions of $\psi$, in equation (20) the field $\psi(0)$ must be given by $(1 / 2)\left(\psi\left(0^{+}\right)+\psi\left(0^{-}\right)\right)$. With this definition, it is straightforward to solve the equations of motion. The solutions are given by

$$
\psi(x)= \begin{cases}\sum_{\omega} A_{\omega} e^{i\left(\omega+\omega_{0}\right) x} e^{-i \omega t} & \text { for } x<0 \\ \sum_{\omega} B_{\omega} e^{i\left(\omega+\omega_{0}\right) x} e^{-i \omega t} & \text { for } x>0\end{cases}
$$

and

$$
\psi^{\dagger}(x)= \begin{cases}\sum_{\omega} A_{-\omega}^{\dagger} e^{i\left(\omega-\omega_{0}\right) x} e^{-i \omega t} & \text { for } x<0 \\ \sum_{\omega} B_{-\omega}^{\dagger} e^{i\left(\omega-\omega_{0}\right) x} e^{-i \omega t} & \text { for } x>0\end{cases}
$$

where

$$
B_{\omega}=\frac{\left(1+e^{i \phi(\omega)}\right) A_{\omega}+\left(1-e^{i \phi(\omega)}\right) A_{-\omega}^{\dagger}}{2}
$$

and

$$
e^{i \phi(\omega)}=\frac{i \omega+4 \pi|\Gamma|^{2}}{i \omega-4 \pi|\Gamma|^{2}}
$$

Given the commutation relation for $\psi$, the $A_{\omega}$ satisfy the following commutation relation:

$$
\left\{A_{\omega_{1}}, A_{\omega_{2}}^{\dagger}\right\}=\delta_{\omega_{1}, \omega_{2}} .
$$

These solutions can be interpreted as having an incident particle at energy $\omega$ that scatters into a particle with energy $\omega$ and a hole with energy $-\omega$ (see Fig. 4). Both the particle and hole scattering involve an energy dependent phase shift. 
The reservoir is located to the left of the impurity, for some $x<0$. To obtain the scattering state $|\Phi\rangle$, we assume that the states leaving the reservoir are in equilibrium with the reservoir, which has energy $\omega_{0}$. Thus, for $x<0$, at zero temperature all the states with $\omega \leq \omega_{0}$ are filled. This means that

$$
A_{\omega}^{\dagger}|\Phi\rangle=0 \quad \text { for } \quad \omega<\omega_{0}
$$

and

$$
A_{\omega}|\Phi\rangle=0 \quad \text { for } \quad \omega>\omega_{0}
$$

Using the commutation relations for $A$ in equation 26 , we then find that

$$
\left\langle\Phi\left|A_{\omega_{1}} A_{\omega_{2}}\right| \Phi\right\rangle=0
$$

and

$$
\left\langle\Phi\left|A_{\omega_{1}}^{\dagger} A_{\omega_{2}}\right| \Phi\right\rangle=n_{\omega_{1}} \delta_{\omega_{1}, \omega_{2}}
$$

where

$$
n_{\omega}= \begin{cases}1 & \text { for } \omega<\omega_{0} \\ 0 & \text { for } \omega>\omega_{0}\end{cases}
$$

In this paper, we will just concentrate on the case when $T=0$. However, we can obtain the finite temperature results by replacing $n_{\omega}$ with

$$
n_{\omega}=\frac{1}{e^{\beta\left(\omega-\omega_{0}\right)}+1} .
$$

We can now use the solutions for $\psi$ and the scattering state to solve for the noise in both incoming and outgoing channels. Our calculations will closely follow those by Buttiker in reference [18]. The noise is given by

$$
S\left(\omega ; x_{1}, x_{2}\right)=\int_{-\infty}^{\infty} d t e^{i \omega t}\left\langle\left\{\rho_{-}\left(t, x_{1}\right), \rho_{-}\left(0, x_{2}\right)\right\}\right\rangle
$$

where we take only the connected part of the correlation function, and $x_{1}$ and $x_{2}$ are positive or negative depending on whether the current is evaluated in the incoming or outgoing channel.

We will begin by calculating the noise when $x_{1}=x_{2}$. In this case, both of the currents are evaluated on the same side of the impurity. Because of the time translational invariance of the correlators, the expression for the noise simplifies to

$$
S\left(\omega ; x_{1}, x_{1}\right)=\int_{-\infty}^{\infty} d t\left(e^{i \omega t}+e^{-i \omega t}\right)\left\langle\rho_{-}\left(t, x_{1}\right) \rho_{-}\left(0, x_{1}\right)\right\rangle
$$

To find the noise in the incoming channel, we must evaluate the expectation value

$$
\left\langle\rho_{-}\left(t, x_{-}\right) \rho_{-}\left(0, x_{-}\right)\right\rangle=\left\langle\psi^{\dagger}\left(t, x_{-}\right) \psi\left(t, x_{-}\right) \psi^{\dagger}\left(0, x_{-}\right) \psi\left(0, x_{-}\right)\right\rangle
$$

with $x_{-}<0$. Using the solutions (22) and (23) for $\psi$ and $\psi^{\dagger}$, we find

$$
\left\langle\rho_{-}\left(t, x_{-}\right) \rho_{-}\left(0, x_{-}\right)\right\rangle=\sum_{\omega_{1}, \omega_{2}, \omega_{3}, \omega_{4}} e^{-i\left(\omega_{1}+\omega_{2}\right) t}\left\langle\Phi\left|A_{-\omega_{1}}^{\dagger} A_{\omega_{2}} A_{-\omega_{3}}^{\dagger} A_{\omega_{4}}\right| \Phi\right\rangle e^{i\left(\omega_{1}+\omega_{2}+\omega_{3}+\omega_{4}\right) x_{-}} .
$$

This expectation value, and the resulting integrals for $S\left(\omega ; x_{-}, x_{-}\right)$are evaluated in Appendix C, with the result,

$$
S\left(\omega ; x_{-}, x_{-}\right)=\frac{1}{2 \pi}|\omega| .
$$

If we want to calculate the noise in one of the two original $R$ and $L$ incoming branches, we must use the relations

$$
\rho_{R}(x)=\frac{1}{2}\left(\rho_{+}(x)+\rho_{-}(x)\right) \quad \text { and } \quad \rho_{L}(x)=\frac{1}{2}\left(\rho_{+}(-x)-\rho_{-}(-x)\right)
$$


Then the density-density correlations can be evaluated as follows:

$$
\left\langle\rho_{R, L} \rho_{R, L}\right\rangle=\frac{1}{4}\left\langle\left(\rho_{+} \pm \rho_{-}\right)\left(\rho_{+} \pm \rho_{-}\right)\right\rangle=\frac{1}{4}\left\langle\rho_{-} \rho_{-}\right\rangle+\frac{1}{4}\left\langle\rho_{+} \rho_{+}\right\rangle,
$$

where the last equality follows from the fact that $\rho_{+}$and $\rho_{-}$are decoupled. Recall that $\rho_{+}$is a free field, so that the contribution to the noise from $\rho_{+}$is simply $\frac{1}{2 \pi}|\omega|$. We find that the noise in each of the two incoming $R$ and $L$ branches is given by

$$
\begin{aligned}
S_{11}(\omega)=S_{22}(\omega) & =\frac{1}{4} S\left(\omega ; x_{-}, x_{-}\right)+\frac{1}{4} \frac{|\omega|}{2 \pi} \\
& =\frac{1}{4 \pi}|\omega|,
\end{aligned}
$$

just as we found in the perturbative calculation with $\nu=1 / 2$ in Eqs. (7) to (10). Using this scattering approach, it is clear that for these two incoming probes the noise is the same as for a free system, because in these two channels the densities have not yet reached the impurity.

Next, we will calculate the noise in the outgoing current. This time we must evaluate the correlator

$$
\left\langle\rho_{-}\left(t, x_{+}\right) \rho_{-}\left(0, x_{+}\right)\right\rangle=\left\langle\psi^{\dagger}\left(t, x_{+}\right) \psi\left(t, x_{+}\right) \psi^{\dagger}\left(0, x_{+}\right) \psi\left(0, x_{+}\right)\right\rangle,
$$

with $x_{+}>0$. According to equations 222) and (23), this is equal to

$$
\left\langle\rho_{-}\left(t, x_{+}\right) \rho_{-}\left(0, x_{+}\right)\right\rangle=\sum_{\omega_{1}, \omega_{2}, \omega_{3}, \omega_{4}} e^{-i\left(\omega_{1}+\omega_{2}\right) t}\left\langle\Phi\left|B_{-\omega_{1}}^{\dagger} B_{\omega_{2}} B_{-\omega_{3}}^{\dagger} B_{\omega_{4}}\right| \Phi\right\rangle e^{i\left(\omega_{1}+\omega_{2}+\omega_{3}+\omega_{4}\right) x_{+}} .
$$

When we expand the $B$ 's in terms of the $A$ 's, we will obtain two different types of processes (see Fig. 5). In the first, at time 0 one particle is created while another is destroyed, and then at time $t$ the first particle is destroyed and another is created. In terms of the original tunneling picture, this describes the process where both at time $t$ and at time 0 one quasiparticle tunnels from the left branch to the right branch and another tunnels in the opposite direction. In the second process, at time 0 two particles are created and then at time $t$ they are destroyed (or vice versa). In the original tunneling picture, this corresponds to two quasiparticles tunneling in one direction at time 0 and two quasiparticles tunneling in the opposite direction at time $t$. As shown in Appendix $\mathrm{C}$, this second process is responsible for the electron singularity at $\tilde{\omega}_{0}=2 \omega_{0}$. In Appendix C, the expectation values in Eq. (42) and the integrals for $S\left(\omega ; x_{+}, x_{+}\right)$are evaluated. We find that the noise on the outgoing side of the impurity is

$$
\begin{aligned}
S\left(\omega ; x_{+}, x_{+}\right)(\omega)=\frac{1}{2 \pi}|\omega|+ & \theta\left(\left|2 \omega_{0}\right|-|\omega|\right)\left\{4|\Gamma|^{2}\left[\tan ^{-1}\left(\frac{\left|\omega_{0}\right|}{4 \pi|\Gamma|^{2}}\right)+\tan ^{-1}\left(\frac{\left|\omega_{0}\right|-|\omega|}{4 \pi|\Gamma|^{2}}\right)\right]\right. \\
+ & \left.\frac{16 \pi|\Gamma|^{4}}{|\omega|}\left[\ln \left(\left(4 \pi|\Gamma|^{2}\right)^{2}+\left(|\omega|-\left|\omega_{0}\right|\right)^{2}\right)-\ln \left(\left(4 \pi|\Gamma|^{2}\right)^{2}+\omega_{0}^{2}\right)\right]\right\} .
\end{aligned}
$$

To compare with our perturbative calculation for the noise in the original four probe geometry, we again make use of equation (39). Thus, the noise in the two outgoing branches is related to $S\left(\omega ; x_{+}, x_{+}\right)$as follows:

$$
S_{33}(\omega)=S_{44}(\omega)=\frac{1}{4} S\left(\omega ; x_{+}, x_{+}\right)+\frac{1}{4} \frac{|\omega|}{2 \pi} .
$$

The first striking feature to note in equation (43) is that the noise due to the tunneling vanishes identically for $|\omega|>\left|2 \omega_{0}\right|$. This means that whenever $|\omega|$ is larger than the electron frequency, the noise shows no sign of the impurity; it is the same as for the incoming branch. This is also what happens for the free electron case, with $g=1$. To second order in perturbation theory, this is indeed the case for any $g$, as seen in the previous section. The strength of the results presented here is that for any value of the coupling $\Gamma$ the noise vanishes above the electron frequency when $g=1 / 2,1$ and 2. (The last case, $g=2$, is obtained by resorting to the strong coupling limit of the $g=1 / 2$ case.) It is not clear whether this will happen for the other values of $g$ beyond second order in perturbation theory.

Next, we can expand $S\left(\omega ; x_{+}, x_{+}\right)$out for small and large $|\Gamma|$ to compare with the perturbative results. As $|\Gamma|$ goes to zero, the noise becomes

$$
S_{33}(\omega)=S_{44}(\omega)=\frac{1}{4 \pi}|\omega|+\pi|\Gamma|^{2} \theta\left(\left|\omega_{0}\right|-|\omega|\right) .
$$


This agrees with the perturbative result for $g=1 / 2$. We note that the quasiparticle singularity arises because we took the $|\Gamma| \rightarrow 0$ limit of the arctangents. In addition, because this step function is already zero for $|\omega|>\left|\omega_{0}\right|$, the electron singularity at $|\omega|=\left|2 \omega_{0}\right|$ drops out. Thus, to this order we only have the quasiparticle singularity. However, for any finite value of $|\Gamma|$ the quasiparticle singularity becomes smoothed out and the electron singularity appears. As we shall see later, though, the "smoothed out" quasiparticle singularity is still a more distinctive feature in the plots of the full noise than is the electron singularity.

Next, for $|\Gamma| \rightarrow \infty$, the noise becomes

$$
S_{33}(\omega)=S_{44}(\omega)=\frac{1}{4 \pi}|\omega|+\frac{1}{384 \pi^{3}|\Gamma|^{4}} \theta\left(\left|2 \omega_{0}\right|-|\omega|\right)\left(\left|2 \omega_{0}\right|-|\omega|\right)^{3}+O\left(1 /|\Gamma|^{8}\right) .
$$

If we make the identification that $\Gamma_{\frac{1}{2}}$, the tunneling amplitude for $g=1 / 2$, is related to $\Gamma_{2}$, the tunneling amplitude for $g=2$, by

$$
\left|\Gamma_{2}\right|=\frac{1}{16 \pi^{2}\left|\Gamma_{\frac{1}{2}}\right|^{2}}
$$

then this answer agrees with the perturbative result for $g=2$. (To make the comparison, we must recall that the $\omega_{0}$ in this equation corresponds to the Josephson frequency for the quasiparticle, whereas the $\omega_{J}$ in the perturbative calculation Eqs. (7) to (10) is the Josephson frequency for the electron, which is twice as large.) In addition, the expansion in $\frac{1}{|\Gamma|}$ of the scattering solution only contains powers of $\frac{1}{|\Gamma|^{4}}=\left|\Gamma_{2}\right|^{2}$, and at every order in $\left|\Gamma_{2}\right|^{2}$ the electron singularity at $|\omega|=\left|2 \omega_{0}\right|$ remains. These two properties also agree with the perturbative results found in 15$]$.

Lastly, we can make use of the scaling properties of the noise to write $\tilde{S}=S / 2|\Gamma|^{2}$ as a function only of $\tilde{\omega}=\frac{\omega}{4 \pi|\Gamma|^{2}}$ and $\tilde{\omega}_{0}=\frac{\omega_{0}}{4 \pi|\Gamma|^{2}}$. The noise is then given by

$$
\begin{aligned}
\tilde{S}_{33}(\tilde{\omega})= & \tilde{S}_{44}(\tilde{\omega}) \\
= & \frac{1}{2}|\tilde{\omega}|+\theta\left(\left|2 \tilde{\omega}_{0}\right|-|\tilde{\omega}|\right)\left\{\frac{1}{2}\left[\tan ^{-1}\left(\left|\tilde{\omega}_{0}\right|\right)+\tan ^{-1}\left(\left|\tilde{\omega}_{0}\right|-|\tilde{\omega}|\right)\right]\right. \\
& \left.\quad+\frac{1}{2|\tilde{\omega}|}\left[\ln \left(1+\left(|\tilde{\omega}|-\left|\tilde{\omega}_{0}\right|\right)^{2}\right)-\ln \left(1+\tilde{\omega}_{0}^{2}\right)\right]\right\} .
\end{aligned}
$$

In Figure 6a, the excess noise $\tilde{S}-\tilde{S}^{\tilde{\omega}_{0}=0}$ is plotted against $\tilde{\omega} / \tilde{\omega}_{0}$ for different values of $\tilde{\omega}_{0}$. As $\tilde{\omega}_{0}$ becomes large, the excess noise approaches the step function in Eq. (45). Recall that $\tilde{\omega}_{0}=\tilde{\omega} /\left(4 \pi|\Gamma|^{2}\right)$, so this limit is equal to the weak coupling limit with $|\Gamma| \rightarrow 0$. To see the strong coupling limit, in Fig. $6 \mathrm{~b}$ we plot the excess noise divided by $\tilde{\omega}_{0}^{3}$ (in order to fit in the same scale). As $\tilde{\omega}_{0}$ becomes small, this clearly has the cubic behavior in Eq. (46). Finally, the full noise, divided by $\tilde{\omega}_{0}$, is plotted in Fig. 6c. The cubic singularity at $\tilde{\omega}=2 \tilde{\omega}_{0}$ decays too quickly to appear in the full noise. However, for $\tilde{\omega}_{0}=100$ and $\tilde{\omega}_{0}=10$, there is clearly a "blip" in the plot of the noise, which shows the "smoothed out" quasiparticle singularity.

For completeness, we will conclude this section by giving the result for the noise $S\left(\omega ; x_{+}, x_{-}\right)$between incoming and outgoing currents. By comparing this with the perturbative calculations of the cross-correlations, we will see to what extent the duality symmetry holds. In addition, once we have $S\left(\omega ; x_{+}, x_{-}\right), S\left(\omega ; x_{+}, x_{+}\right)$and $S\left(\omega ; x_{-}, x_{-}\right)$, we can calculate the noise in the Hall current and the tunneling current. The Hall current is the total current running down the sample, given by $I_{H}=j_{L}(x)+j_{R}(x)=\rho_{R}(x)-\rho_{L}(x)$ and the tunneling current is the current that tunnels across the sample, which is given by $I_{t}=\rho_{R}\left(x_{+}\right)-\rho_{R}\left(x_{-}\right)=\rho_{L}\left(x_{-}\right)-\rho_{L}\left(x_{+}\right)$.

The expression for the $S\left(\omega ; x_{+}, x_{-}\right)$noise is

$$
S\left(\omega ; x_{+}, x_{-}\right)=\int_{-\infty}^{\infty} d t e^{i \omega t}\left\langle\left\{\rho_{-}\left(t, x_{+}\right), \rho_{-}\left(0, x_{-}\right)\right\}\right\rangle
$$

where $x_{-}<0$ and $x_{+}>0$. Again, we can expand the $\rho_{-}\left(x_{+}\right)$and $\rho_{-}\left(x_{-}\right)$in terms of the $A_{\omega}$ 's and $B_{\omega}$ 's in the solution for $\psi$. After evaluating the expectation values and performing the integrals over $\omega_{i}$ and $t$, we find

$$
\begin{gathered}
S\left(\omega ; x_{+}, x_{-}\right)=\left\{\frac{|\omega|}{2 \pi}-2|\Gamma|^{2}\left[\tan ^{-1}\left(\frac{|\omega|-\omega_{0}}{4 \pi|\Gamma|^{2}}\right)+\tan ^{-1}\left(\frac{|\omega|+\omega_{0}}{4 \pi|\Gamma|^{2}}\right)\right]\right. \\
+i|\Gamma|^{2} \operatorname{sign}(\omega)\left[2 \ln \left(\omega_{0}^{2}+\left(4 \pi|\Gamma|^{2}\right)^{2}\right)-\ln \left(\left(\omega+\omega_{0}\right)^{2}+\left(4 \pi|\Gamma|^{2}\right)^{2}\right)\right. \\
\left.\left.-\ln \left(\left(\omega-\omega_{0}\right)^{2}+\left(4 \pi|\Gamma|^{2}\right)^{2}\right)\right]\right\} e^{i \omega\left(x_{+}-x_{-}\right)} .
\end{gathered}
$$


We can again use equation (39) to obtain the expression for the cross-correlations of the currents in the original four reservoirs. We find, for example,

$$
S_{31}(\omega)=\frac{1}{4} S\left(\omega ; x_{+}, x_{-}\right)+\frac{1}{4} \frac{|\omega|}{2 \pi} e^{i \omega\left(x_{+}-x_{-}\right)} .
$$

and

$$
S_{41}(\omega)=-\frac{1}{4} S\left(\omega ; x_{+}, x_{-}\right)+\frac{1}{4} \frac{|\omega|}{2 \pi} e^{i \omega\left(x_{+}-x_{-}\right)} .
$$

The other cross-correlations, namely $S_{32}$ and $S_{42}$, can be calculated similarly. For small $|\Gamma|$, the noise is

$$
\begin{aligned}
S_{31}(\omega)=\left[\frac{|\omega|}{4 \pi}\right. & -\frac{\pi}{4}|\Gamma|^{2}\left(\operatorname{sign}\left(|\omega|+\omega_{0}\right)+\operatorname{sign}\left(|\omega|-\omega_{0}\right)\right) \\
& \left.-i \frac{1}{2}|\Gamma|^{2} \ln \left(\left|\frac{\omega^{2}-\omega_{0}^{2}}{\omega_{0}^{2}}\right|\right)\right] e^{i \omega\left(x_{+}-x_{-}\right)}
\end{aligned}
$$

and when $|\Gamma|$ is large, the noise becomes

$$
\begin{aligned}
S_{41}(\omega)=\left\{\frac{|\omega|}{4 \pi}\right. & +i \frac{1}{32 \pi^{2}} \frac{1}{|\Gamma|^{2}} \omega^{2} \operatorname{sign}(\omega) \\
& \left.-\frac{1}{384 \pi^{3}} \frac{1}{|\Gamma|^{4}}\left[\left(|\omega|+\omega_{0}\right)^{3}+\left(|\omega|-\omega_{0}\right)^{3}\right]\right\} e^{i \omega\left(x_{+}-x_{-}\right)}
\end{aligned}
$$

In the following section, we will compare these results with the perturbative calculation. We will find that for $g=1 / 2$ they agree, but they differ for $g=2$. In Section V, we will also discuss this apparent breakdown of the duality transformation.

\section{DISCUSSION OF THE DUALITY SYMMETRY}

As we have seen in the previous sections, we expect this system to exhibit a duality symmetry. In this section, we will first describe this duality symmetry more fully, and then compare the results from the perturbative and scattering calculation to see how consistent they are with this symmetry.

For $g=1 / 2$, the original picture of this system is a single quantum Hall droplet with "filling fraction" $\nu=1 / 2$. Quasiparticles can tunnel from one branch to the other, and they have charge $e^{*}=\nu e$, tunneling amplitude $\Gamma_{q}$, and Josephson frequency $\omega_{0}=e^{*} V / \hbar$. The Lagrangian describing this system can be written as

$$
\mathcal{L}=\frac{1}{8 \pi}\left[\left(\partial_{t} \phi\right)^{2}-v^{2}\left(\partial_{x} \phi\right)^{2}\right]-\Gamma_{q} e^{-i \omega_{0} t} \delta(x) e^{i \sqrt{g} \phi(t, 0)}+H . c .,
$$

with $g=1 / 2$ and $\phi=\phi_{R}(x)+\phi_{L}(-x)$. If we use the four probe geometry to study this system, then Eq. (5) gives the relation between the densities in the four probes, $\rho_{1}, \rho_{2}, \rho_{3}$, and $\rho_{4}$, and the densities of the leftmovers and rightmovers. They are shown in Fig. 7a.

Once $\Gamma$ is increased (or $V$ is decreased), the droplet should split into two. Each of the two new droplets is still characterized by filling fraction $\nu$. However, now only electrons can tunnel across the gap from one branch to the other. For $g=1 / 2$, the electron is made up of two quasiparticles, so the tunneling operator for the electron should be

$$
\Gamma_{e}\left(e^{i \sqrt{1 / 2} \phi(t, 0)}\right)^{2}=\Gamma_{e} e^{i \sqrt{2} \phi(t, 0)}
$$

the charge is $e$, and the Josephson frequency is $\tilde{\omega}_{0}=2 \omega_{0}$. Thus, when $\Gamma_{q}$ becomes large, this system can also be described by the Lagrangian density

$$
\mathcal{L}=\frac{1}{8 \pi}\left[\left(\partial_{t} \phi\right)^{2}-v^{2}\left(\partial_{x} \phi\right)^{2}\right]-\Gamma_{e} e^{-i \tilde{\omega}_{0} t} \delta(x) e^{i \sqrt{g} \phi(t, 0)}+H . c .,
$$

where $\tilde{g}=2$. However, in this geometry with the two droplets, we must be careful when we write the densities in the four probes in terms of the left-moving and right-moving densities. According to Fig. $7 \mathrm{~b}$, this relation is given by 


$$
\begin{array}{lll}
\rho_{1}(t)=\rho_{R}(t, \tilde{x}) & \text { for } & \tilde{x}<0 \\
\rho_{2}(t)=\rho_{L}(t, \tilde{x}) & \text { for } & \tilde{x}>0 \\
\rho_{3}(t)=\rho_{L}(t, \tilde{x}) & \text { for } & \tilde{x}<0 \\
\rho_{4}(t)=\rho_{R}(t, \tilde{x}) & \text { for } & \tilde{x}>0 .
\end{array}
$$

With these identifications, $S_{31}(\omega)$ in the four probe geometry equals $S_{L R}\left(\omega ; \tilde{x}_{-}, \tilde{x}_{-}\right)$in the two-droplet geometry, and similarly, $S_{41}(\omega)$ is given by $S_{R R}\left(\omega ; \tilde{x}_{+}, \tilde{x}_{-}\right)$, where $\tilde{x}_{-}<0$ and $\tilde{x}_{+}>0$. Also, we see that the Hall current in the single droplet, $\rho_{R}(t, x)-\rho_{L}(t, x)$ is dual to the tunneling current in the two droplets, $\rho_{R}\left(t, \tilde{x}_{-}\right)-\rho_{R}\left(t, \tilde{x}_{+}\right)$, because both are equivalent to $\rho_{1}(t)-\rho_{4}(t)$.

We will first verify that the scattering and perturbative calculations agree for $g=1 / 2$. We have already found that when the noise is evaluated on only one side of the junction, then both the scattering and perturbative results agree. If one probe is in an incoming channel and the other probe is in an outgoing channel, then according to Appendix A the perturbative result for the noise is

$$
\begin{aligned}
S_{31}\left(\omega ; x_{+}, x_{-}\right)=e^{i \omega\left(x_{+}-x_{-}\right)}\{ & \frac{|\omega|}{4 \pi} \\
& \left.-\frac{\left|\Gamma_{q}\right|^{2}}{8}\left[i 4 \operatorname{sign}(\omega) \ln \left(\left|\frac{\omega^{2}-\omega_{0}^{2}}{\omega_{0}^{2}}\right|\right)+2 \pi\left[\left(1+\operatorname{sign}\left(|\omega|-\left|\omega_{0}\right|\right)\right)\right]\right]\right\},
\end{aligned}
$$

where we have set $g=\nu=1 / 2$ in Eq. (A19). On comparing this with the expansion of the scattering calculation for small $\Gamma$ in Eq. (53), we find that also in this case the scattering and perturbative results agree.

Next, to check the duality transformation, we must compare the scattering calculation as $\Gamma \rightarrow \infty$ with the perturbative calculation at $g=2$. Again we found that if both probes are in the same branch then the two calculations agree. This is rather remarkable, because when $g=2$ the system can be sensitive to short distance behavior, which means that it could depend on the detailed structure of the junction and on how it is regulated. However, here we found that the weak-coupling perturbative calculation and the strong-coupling limit of the scattering calculation are the same, even though they treat the junction very differently. We conclude that, at least to the order in perturbation theory that we have calculated, the noise extracted from a single channel is not affected by the short-distance properties of the impurity.

To complete the comparison, we need the results for the noise between the incoming and outgoing channels. Using Eq. (47) to relate the quasiparticle tunneling amplitude to the electron tunneling amplitude, we find that the expansion for $\Gamma \rightarrow \infty$ of the scattering calculation becomes

$$
S_{41}\left(\omega ; x_{+}, x_{-}\right)=\left\{\frac{1}{4 \pi}|\omega|+\frac{i}{2}\left|\Gamma_{e}\right| \omega^{2} \operatorname{sign}(\omega)-\frac{2 \pi}{3}\left|\Gamma_{e}\right|^{2}\left[\left(|\omega|+\omega_{0}\right)^{3}+\left(|\omega|-\omega_{0}\right)^{3}\right]\right\} e^{i \omega\left(x_{+}-x_{-}\right)} .
$$

This must be compared with the perturbative calculation of $S_{R R}\left(\omega ; \tilde{x}_{+}, \tilde{x}_{-}\right)$. To obtain this perturbative result, we set $\nu=1 / 2, g=2$ and replace $\omega_{0}$ by $2 \omega_{0}$ in Eq. (A19). Then the perturbative calculation of the noise across the junction yields

$$
S^{R R}\left(\omega ; \tilde{x}_{+}, \tilde{x}_{-}\right)=\left\{\frac{1}{4 \pi}|\omega|-\left|\Gamma_{e}\right|^{2}\left[\frac{\pi}{6}\left(\left(|\omega|+\left|2 \omega_{0}\right|\right)^{3}+\left(|\omega|-\left|2 \omega_{0}\right|\right)^{3}\right)+\frac{2 i \omega^{2}}{3 \delta}\right]\right\} e^{i \omega\left(x_{+}-x_{-}\right)} .
$$

We first note that this expression for the noise contains a linear divergence in the cutoff $\delta$. Thus this perturbative calculation is regulator dependent, which is not surprising because the tunneling operator at $g=2$ has dimension 2 and should be an irrelevant operator. In spite of this, both calculations do agree to order $\omega$ (which is all that the derivations of the duality transformation in reference [13] would predict); it is only the higher order terms in $\omega$ that disagree. This suggests that we are on the right track with the perturbative calculation, but we just need to add in the appropriate counter terms.

To see which counter term we should add, we begin by recalling that we used the most relevant tunneling operator to describe the system. However, for $g=2$ the operators $\left(\rho_{L}\right)^{2}+\left(\rho_{R}\right)^{2}$ and $\rho_{L} \rho_{R}$ are just as relevant as the tunneling operator, so we must consider their effects also. In fact, $\left(\rho_{R}(0)-\rho_{L}(0)\right)^{2}$ also encourages tunneling because it tries to equalize the density of right movers and left movers. Another way to look at it is that we cannot have quasiparticles tunneling between the droplets, but density fluctuations on one side may affect the other side.

In Appendix B, we found that when the interaction 


$$
\mathcal{L}_{\text {int }}=\gamma\left(\rho_{R}(t, 0)-\rho_{L}(t, 0)\right)^{2} \delta(x)
$$

is included in the Lagrangian, it gives the following contribution to the noise

$$
S_{\rho \rho}^{R R}\left(x_{1}, x_{2}\right)=\theta\left(-x_{1} x_{2}\right)\left\{\frac{i \gamma}{8 \pi^{2}} \omega^{2} \operatorname{sign}\left(\omega x_{1}\right)-\frac{\gamma^{2}}{16 \pi^{3}}\left[|\omega|^{3}-2 i^{\prime \prime} \delta(0)^{\prime \prime} \omega^{2} \operatorname{sign}\left(\omega x_{1}\right)\right]\right\} e^{i \omega\left(x_{1}-x_{2}\right)} .
$$

First, we note that the density-density coupling does not affect the noise evaluated on only one side of the impurity (i.e. when $x_{1} x_{2}>0$.) According to equations (7) to (10), (46), and (47), this is necessary for the scattering and the perturbative calculations to agree. It is reasonable that the noise evaluated on only one side of the junction should be less affected by the counter terms and the regulator than the noise between probes on either side of the junction, because even though in both cases all the measurements are done far from the junction, in the second case the information must travel from one side of the junction to the other.

Second, we note that when $x_{1} x_{2}<0$, equation (63) contains the linear term in $\gamma$, which also appears in the scattering calculation, but not in the original perturbative calculation. We find that the only density-density interaction that gives the same linear term as in the scattering result for all of the cross-correlations is the one given in equation (62), with $\gamma=1 /\left(4 \Gamma_{q}^{2}\right)$. When we add the density-density term with this choice for $\gamma$ to the original perturbative calculation, we obtain

$$
\begin{aligned}
S_{\text {pert }}^{R R}\left(\omega ; x_{+}, x_{-}\right)=\left\{\frac{1}{4 \pi}|\omega|\right. & +\frac{i}{2}\left|\Gamma_{e}\right| \omega^{2} \operatorname{sign}(\omega)-\frac{2 \pi}{3}\left|\Gamma_{e}\right|^{2}\left[\left(|\omega|+\omega_{0}\right)^{3}+\left(|\omega|-\omega_{0}\right)^{3}\right] \\
& \left.+2 i\left|\Gamma_{e}\right|^{2} \omega^{2} \operatorname{sign}(\omega)\left(\pi “ \delta(0)^{\prime \prime}-\frac{1}{3 \delta}\right)\right\} e^{i \omega\left(x_{+}-x_{-}\right)} .
\end{aligned}
$$

Thus, (except for the divergent part), this perturbative result agrees with the scattering result.

To cancel the divergent part, we must regulate the delta-function properly and adjust the counterterm accordingly. Then the two results will agree in the limit as $x_{+}$and $x_{-} \rightarrow \pm \infty$. Another approach, which may be more appropriate, is to "smooth out" the density-density interaction. This is accomplished by replacing the interaction in equation (62) by the following expression

$$
\mathcal{L}_{\text {int }}=\gamma\left(\rho_{R}(t, 0)-\rho_{L}(t, 0)\right)^{2} f_{\epsilon}(x),
$$

where $f_{\epsilon}(x) \rightarrow \delta(x)$ as $\epsilon \rightarrow 0$. This new interaction does not change the finite part of Eq. (64), and the function $f$ can be chosen so that the divergence cancels. As a result, even though the duality symmetry is not exactly obeyed for the cross-correlations, it is possible to add in counter terms to bring the strong-coupling limit of one picture into agreement with the weak-coupling limit of the dual picture.

To summarize, to the order in $\Gamma$ we have calculated, the action for $g=1 / 2$ is dual to the renormalized action for $g=2$, given by

$$
\mathcal{L}=\frac{1}{8 \pi}\left[\left(\partial_{t} \phi\right)^{2}-v^{2}\left(\partial_{x} \phi\right)^{2}\right]-\Gamma_{e} e^{-i 2 \omega_{0} t} \delta(x) e^{\sqrt{2} \phi(t, 0)}+H . c .+4 \pi^{2} \Gamma_{e} \delta(x)\left(\rho_{R}(t, 0)-\rho_{L}(t, 0)\right)^{2} ;
$$

and if we only want to calculate the noise in one particular channel, then it is not necessary to include the $\rho \rho$ interaction to obtain the dual picture. As explained above, this action can be interpreted as containing two different terms that induce or encourage tunneling. We can also use the relation

$$
\rho_{R}(0)-\rho_{L}(0)=\frac{1}{2 \sqrt{2} \pi} \partial_{x} \phi(0)
$$

to write the action as

$$
\mathcal{L}=\frac{1}{8 \pi}\left[\left(\partial_{t} \phi\right)^{2}-\tilde{v}^{2}\left(\partial_{x} \phi\right)^{2}\right]-\Gamma_{e} e^{-i 2 \omega_{0} t} \delta(x) e^{\sqrt{\tilde{2}} \phi(t, 0)}+\text { H.c. },
$$

where $\tilde{v}^{2}=v^{2}+4 \pi \delta(x) \Gamma_{e}^{2}$ is the "renormalized" velocity. In this case, the velocity remains the same everywhere but right at the junction. If, instead, we use Eq. 65) for the density interaction, then the velocity is renormalized in a region around the junction. 


\section{CONCLUSION}

In this paper we studied the four terminal tunneling noise spectrum for chiral Luttinger liquids characterized by an exponent $g$. Perturbative results are obtained for arbitrary $g$. Perturbative calculations for quasiparticle tunneling reveal a singularity at the quasiparticle Josephson frequency $\nu \mathrm{eV} / \hbar$, while perturbative calculations for electron tunneling only produce a singularity at the electron Josephson frequency $e V / \hbar$. This appears to be inconsistent with the duality picture that quasiparticle and electron tunneling describe the same tunneling junction in two different limits. To understand how the quasiparticle tunneling picture can smoothly connect to the electron tunneling picture, we calculated the exact noise spectrum for $g=1 / 2$ (or $g=2$ due to duality). We find that the singularity at the quasiparticle Josephson frequency $\frac{1}{2} \mathrm{eV} / \hbar$ is smeared for finite tunneling and is not a true singularity, while the singularity at the electron Josephson frequency $\mathrm{eV} / \hbar$ survives in the exact result. From the exact result we also find that the noise spectrum of the current correlations on a single branch (auto-correlations) satisfies the duality relation, while current correlations between distinct branches (cross-correlations) do not satisfy the naive duality relation.

\section{ACKNOWLEDGEMENTS}

We would like to thank Akira Furusaki for helpful discussions. This work is supported by NSF grants DMR-9400334 (CCC) and DMR-9411574 (XGW). XGW acknowledges the support from the A.P. Sloan Foundation. D. F. is currently a Bunting Fellow sponsored by the Office of Naval Research and is also supported in part by funds provided by the U. S. Department of Energy (D.O.E.) under cooperative agreement \#DE-AC02-76ER03069 and by National Science Foundation grant PHY9218167.

\section{APPENDIX A: PERTURBATIVE CALCULATION}

In order to obtain the noise spectrum of density-density correlations on given leads, we start by writing the correlations between density operators as follows:

$$
\left\langle\rho_{a}\left(t, x_{1}\right) \rho_{b}\left(0, x_{2}\right)\right\rangle
$$

where $a, b$ take the values +1 for $R$ moving branches and -1 for $L$ moving ones. Such compressed notation makes it simpler to identify incoming and outgoing branches in a unified way for both left and right movers: $\rho_{a}\left(t, x_{1}\right)$, for example, is the density in an incoming or outgoing branch if $a x_{1}<0$ or $a x_{1}>0$, respectively.

The densities are related to the fields $\phi_{R, L}$ through $\rho_{R, L}=\frac{\sqrt{\nu}}{2 \pi} \partial_{x} \phi_{R, L}$, so that we can write

$$
\left\langle\rho_{a}\left(t, x_{1}\right) \rho_{b}\left(t^{\prime}, x_{2}\right)\right\rangle=\frac{\nu}{(2 \pi)^{2}} \partial_{x_{1}} \partial_{x_{2}}\left\langle\phi_{a}\left(t, x_{1}\right) \phi_{b}\left(t^{\prime}, x_{2}\right)\right\rangle,
$$

where it is convenient to use

$$
\left\langle\phi_{a}\left(t, x_{1}\right) \phi_{b}\left(t^{\prime}, x_{2}\right)\right\rangle=\left.\frac{d}{d \lambda_{1}} \frac{d}{d \lambda_{2}}\left\langle e^{i \lambda_{1} \phi_{a}\left(t, x_{1}\right)} e^{-i \lambda_{2} \phi_{b}\left(t^{\prime}, x_{2}\right)}\right\rangle\right|_{\lambda_{1}, \lambda_{2}=0} .
$$

The last correlation function is easy to calculate perturbatively using

$$
\left\langle T_{c}\left(e^{i \lambda_{1} \phi_{a}\left(t, x_{1}\right)} e^{-i \lambda_{2} \phi_{b}\left(t^{\prime}, x_{2}\right)}\right)\right\rangle=\left\langle 0\left|T_{c}\left(S(-\infty,-\infty) e^{i \lambda_{1} \phi_{a}\left(t, x_{1}\right)} e^{-i \lambda_{2} \phi_{b}\left(t^{\prime}, x_{2}\right)}\right)\right| 0\right\rangle,
$$

where $|0\rangle$ is the unperturbed ground state, and $T_{c}$ is the ordering along the Keldysh contour (Fig. 8). The scattering operator $S(-\infty,-\infty)$ takes the initial state, evolves it from $t=-\infty$ to $t=\infty$ and back to $t=-\infty$. The use of the Keldysh contour is necessary in the treatment of non- equilibrium problems, such as the one we have in hand. A more detailed description of the method in the context treated here can be found in Ref. [15].

In order to proceed we expand $S(-\infty,-\infty)$ to second order in perturbation theory. In terms of the Coulomb gas of Ref. [15], we have an insertion of two charges of opposite sign:

$$
\begin{aligned}
& \left\langle T_{c}\left(e^{i \lambda_{1} \phi_{a}\left(t, x_{1}\right)} e^{-i \lambda_{2} \phi_{b}\left(t^{\prime}, x_{2}\right)}\right)\right\rangle_{|\Gamma|^{2}}= \\
& \quad(i \Gamma)\left(i \Gamma^{*}\right) \oint_{c} d t_{+} \oint_{c} d t_{-} e^{i \omega_{0} t_{+}} e^{-i \omega_{0} t_{-}}\left\langle 0\left|T_{c}\left(e^{i q \phi\left(t_{+}, 0\right)} e^{-i q \phi\left(t_{-}, 0\right)} e^{i \lambda_{1} \phi_{a}\left(t, x_{1}\right)} e^{-i \lambda_{2} \phi_{b}\left(t^{\prime}, x_{2}\right)}\right)\right| 0\right\rangle,
\end{aligned}
$$


where $q=\sqrt{g}$, and $\phi$ without subscript stands for the sum $\phi_{R}+\phi_{L}$. The expression above is simplified using

$$
\left\langle 0\left|T_{c}\left(\prod_{j} e^{i q_{j} \phi\left(t_{j}, x_{j}\right)}\right)\right| 0\right\rangle=e^{-\sum_{i>j} q_{i} q_{j}\left\langle 0\left|T_{c}\left(\phi\left(t_{i}, x_{i}\right) \phi\left(t_{j}, x_{j}\right)\right)\right| 0\right\rangle} .
$$

Substituting it into Eq. (A3) we obtain

$$
\begin{aligned}
\left\langle T_{c}\left(\phi_{a}\left(t, x_{1}\right) \phi_{b}\left(t^{\prime}, x_{2}\right)\right)\right\rangle_{|\Gamma|^{2}=\mid}=|\Gamma|^{2} \oint d t_{+} \oint d t_{-} e^{q^{2}\left\langle 0\left|T_{c}\left(\phi\left(t_{+}, 0\right) \phi\left(t_{-}, 0\right)\right)\right| 0\right\rangle} e^{i \omega_{0}\left(t_{+}-t_{-}\right)} \\
\times\left\{q^{2}\left[\left\langle 0\left|T_{c}\left(\phi\left(t_{+}, 0\right) \phi_{a}\left(t, x_{1}\right)\right)\right| 0\right\rangle-\left\langle 0\left|T_{c}\left(\phi\left(t_{-}, 0\right) \phi_{a}\left(t, x_{1}\right)\right)\right| 0\right\rangle\right]\right. \\
\times\left[\left\langle 0\left|T_{c}\left(\phi\left(t_{+}, 0\right) \phi_{b}\left(t^{\prime}, x_{2}\right)\right)\right| 0\right\rangle-\left\langle 0\left|T_{c}\left(\phi\left(t_{-}, 0\right) \phi_{b}\left(t^{\prime}, x_{2}\right)\right)\right| 0\right\rangle\right] \\
\left.+\left\langle 0\left|T_{c}\left(\phi_{a}\left(t, x_{1}\right) \phi_{b}\left(t^{\prime}, x_{2}\right)\right)\right| 0\right\rangle\right\} .
\end{aligned}
$$

The last term in the expression above, the one proportional to $\left.\left\langle 0\left|T_{c}\left(\phi_{a}\left(t, x_{1}\right) \phi_{b}\left(t^{\prime}, x_{2}\right)\right)\right| 0\right\rangle\right\}$, vanishes. The reason why this happens is very simple: the factor in front of it is the term of order $|\Gamma|^{2}$ in the expansion of $Z=\langle 0|S(-\infty,-\infty)| 0\rangle$; since $Z \equiv 1$, the correction at any order in $\Gamma$ must vanish.

In order to carry out the calculations, we introduce notation that keeps track of the position of the two inserted charges along the contour, i.e., whether they are in the forward (or top) branch, or in the return (or bottom) branch (see Fig. 8). The position of the charges is important for the computation of the contour-ordered correlation function, given by $\left\langle 0\left|T_{c}\left(\phi_{R, L}\left(t_{1}, x_{1}\right) \phi_{R, L}\left(t_{2}, x_{2}\right)\right)\right| 0\right\rangle$

$$
= \begin{cases}-\ln \left(\delta+i \operatorname{sign}\left(t_{1}-t_{2}\right)\left[\left(t_{1}-t_{2}\right) \mp\left(x_{1}-x_{2}\right)\right],\right. & \text { both } t_{1} \text { and } t_{2} \text { in the top branch } \\ -\ln \left(\delta-i \operatorname{sign}\left(t_{1}-t_{2}\right)\left[\left(t_{1}-t_{2}\right) \mp\left(x_{1}-x_{2}\right)\right],\right. & \text { both } t_{1} \text { and } t_{2} \text { in the bottom branch } \\ -\ln \left(\delta-i\left[\left(t_{1}-t_{2}\right) \mp\left(x_{1}-x_{2}\right)\right]\right), & t_{1} \text { in the top and } t_{2} \text { in the bottom branch } \\ -\ln \left(\delta+i\left[\left(t_{1}-t_{2}\right) \mp\left(x_{1}-x_{2}\right)\right]\right), & t_{1} \text { in the bottom and } t_{2} \text { in the top branch. }\end{cases}
$$

The compact notation consists of giving indices to the times which contain the information about which branch of the Keldysh contour they are on, so that $t^{\mu}$ is on the top branch for $\mu=+1$, and on the bottom for $\mu=-1$. In this way, we can compress the correlations to a compact form:

$$
\begin{aligned}
G_{\mu \nu}^{a b}\left(t_{1}, x_{1} ; t_{2}, x_{2}\right) & =G_{\mu \nu}^{a b}\left(t_{1}-t_{2}, x_{1}-x_{2}\right)=\left\langle 0\left|T_{c}\left(\phi_{a}\left(t_{1}^{\mu}, x_{1}\right) \phi_{b}\left(t_{2}^{\nu}, x_{2}\right)\right)\right| 0\right\rangle \\
& =-\delta_{a, b} \ln \left(\delta+i K_{\mu \nu}\left(t_{1}-t_{2}\right)\left[\left(t_{1}-t_{2}\right)-a\left(x_{1}-x_{2}\right)\right]\right),
\end{aligned}
$$

where

$$
K_{\mu \nu}(t)=\theta(\mu \nu) \operatorname{sign}(\nu t)+\theta(-\mu \nu) \operatorname{sign}(\nu) .
$$

Again, we have used $a, b= \pm 1$ for $R$ and $L$ fields, respectively. The correlation in Eq. (A7) can be written, using this compressed notation, as

$$
\begin{aligned}
\left\langle T_{c}\left(\phi_{a}\left(t, x_{1}\right) \phi_{b}\left(t^{\prime}, x_{2}\right)\right)\right\rangle_{|\Gamma|^{2}}=\mid & \left.\Gamma\right|^{2} q^{2} \sum_{\mu \nu} \operatorname{sign}(\mu \nu) \int_{-\infty}^{\infty} d t_{+} \int_{-\infty}^{\infty} d t_{-} e^{i \omega_{0}\left(t_{+}-t_{-}\right)} P_{\mu \nu}\left(t_{+}-t_{-}\right) \\
\times & \times\left[G_{+\mu}^{a a}\left(t-t_{+}, x_{1}\right)-G_{+\nu}^{a a}\left(t-t_{-}, x_{1}\right)\right] \\
& \times\left[G_{+\mu}^{b b}\left(t^{\prime}-t_{+}, x_{2}\right)-G_{+\nu}^{b b}\left(t^{\prime}-t_{-}, x_{2}\right)\right]
\end{aligned}
$$

where $P_{\mu \nu}\left(t_{+}-t_{-}\right)=e^{q^{2}\left[G_{\mu \nu}^{++}\left(t_{+}-t_{-}, 0\right)+G_{\mu \nu}^{--}\left(t_{+}-t_{-}, 0\right)\right]}$, or explicitly:

$$
P_{ \pm \pm}(t)=\frac{1}{(\delta \pm i|t|)^{2 g}}, P_{ \pm \mp}(t)=\frac{1}{(\delta \mp i t)^{2 g}} .
$$

The factor $\operatorname{sign}(\mu \nu)$ simply keeps track of the sign coming from the integration of the times $t_{ \pm}$along the contour. Notice that the times $t$ and $t^{\prime}$ are taken to be on the top branch.

Now, let

$$
\begin{aligned}
F_{a b}\left(\omega ; x_{1}, x_{2}\right) & =\int_{-\infty}^{\infty} d t e^{i \omega t}\left\langle T_{c}\left(\rho_{a}\left(t, x_{1}\right) \rho_{b}\left(0, x_{2}\right)\right)\right\rangle_{|\Gamma|^{2}} \\
& =\frac{\nu}{(2 \pi)^{2}} \partial_{x_{1}} \partial_{x_{2}} \int_{-\infty}^{\infty} d t e^{i \omega t}\left\langle T_{c}\left(\phi_{a}\left(t, x_{1}\right) \phi_{b}\left(0, x_{2}\right)\right)\right\rangle_{|\Gamma|^{2}}
\end{aligned}
$$


which can be easily shown, using Eq. (A10), to yield

$$
\begin{aligned}
F_{a b}\left(\omega ; x_{1}, x_{2}\right)=|\Gamma|^{2} \frac{\nu q^{2}}{(2 \pi)^{2}} \sum_{\mu \nu} \operatorname{sign}(\mu \nu) & {\left[\tilde{P}_{\mu \nu}\left(\omega_{0}\right)\left(g_{+\mu}^{a a}\left(\omega, x_{1}\right) g_{+\mu}^{b b}\left(-\omega, x_{2}\right)+g_{+\nu}^{a a}\left(\omega, x_{1}\right) g_{+\nu}^{b b}\left(-\omega, x_{2}\right)\right)\right.} \\
& -\tilde{P}_{\mu \nu}\left(\omega_{0}-\omega\right) g_{+\mu}^{b b}\left(-\omega, x_{2}\right) g_{+\nu}^{a a}\left(\omega, x_{1}\right) \\
& \left.-\tilde{P}_{\mu \nu}\left(\omega_{0}+\omega\right) g_{+\mu}^{a a}\left(\omega, x_{1}\right) g_{+\nu}^{b b}\left(-\omega, x_{2}\right)\right] .
\end{aligned}
$$

In this equation, $g$ is given by $g_{+\mu}^{a a}(\omega, x)=\partial_{x} \tilde{G}_{+\mu}^{a a}(\omega, x)$ and can be obtained from Eqs. (A8) and (A9):

$$
g_{\mu \nu}^{a b}(\omega, x)=\delta_{a, b} \times \begin{cases}\pi i a e^{i \omega a x}(\operatorname{sign}(\omega)+\operatorname{sign}(a x)) & , \mu=+1, \nu=+1 \\ \pi i a e^{i \omega a x}(\operatorname{sign}(\omega)-\operatorname{sign}(a x)) & , \mu=-1, \nu=-1 \\ -2 \pi i a e^{i \omega a x} \theta(-\omega) & , \mu=+1, \nu=-1 \\ 2 \pi i a e^{i \omega a x} \theta(\omega) & , \mu=-1, \nu=+1\end{cases}
$$

The spectrum to second order can be obtained from $F_{a b}\left(\omega, x_{1}, x_{2}\right)$ as follows:

$$
\begin{aligned}
S_{a b}^{(2)}\left(\omega ; x_{1}, x_{2}\right) & =S_{b a}^{(2)}\left(-\omega ; x_{2}, x_{1}\right)=\int_{-\infty}^{\infty} d t e^{i \omega t}\left\langle\left\{\rho_{a}\left(t, x_{1}\right), \rho_{b}\left(0, x_{2}\right)\right\}\right\rangle_{|\Gamma|^{2}} \\
& =F_{a b}\left(\omega ; x_{1}, x_{2}\right)+F_{a b}^{*}\left(-\omega ; x_{1}, x_{2}\right) .
\end{aligned}
$$

The only ingredients remaining to be calculated are the $P(\omega)^{\prime} s$, which are given by:

$$
\begin{aligned}
& \tilde{P}_{++}(\omega)=t(-\omega)=\int_{-\infty}^{\infty} d p \frac{e^{i \omega p}}{(\delta+i|p|)^{2 g}} \\
& \tilde{P}_{--}(\omega)=b(-\omega)=\int_{-\infty}^{\infty} d p \frac{e^{i \omega p}}{(\delta-i|p|)^{2 g}} \\
& \tilde{P}_{ \pm \mp}(\omega)=c_{ \pm}(-\omega)=\int_{-\infty}^{\infty} d p \frac{e^{i \omega p}}{(\delta \mp i p)^{2 g}} .
\end{aligned}
$$

The $t, b, c_{ \pm}$are the same as in Ref. 15. One can easily check that $t(\omega)+b(\omega)=c_{+}(\omega)+c_{-}(\omega)$, and that the $c_{ \pm}$are given by

$$
c_{ \pm}(\omega)=\int_{-\infty}^{\infty} d p \frac{e^{-i \omega p}}{(\delta \mp i p)^{2 g}}=\frac{2 \pi}{\Gamma(2 g)}|\omega|^{2 g-1} e^{-|\omega| \delta} \theta( \pm \omega) .
$$

Now, we have the tools we need in order to obtain all correlations. In particular, correlations within the same branch and taken at the same point, i.e., $a=b$ and $x_{1}=x_{2}$, can be shown to yield:

$$
S_{a a}^{(2)}\left(\omega ; x_{1}, x_{1}\right)=\frac{4 \pi \nu g}{\Gamma(2 g)}|\Gamma|^{2} \theta\left(a x_{1}\right)|| \omega|-| \omega_{0}||^{2 g-1} \theta\left(\left|\omega_{0}\right|-|\omega|\right) .
$$

The zero order term in $\Gamma$ is trivially obtained from the unperturbed density-density correlation functions:

$$
\begin{aligned}
S_{a b}^{(0)}\left(\omega ; x_{1}, x_{2}\right) & =S_{b a}^{(0)}\left(-\omega ; x_{2}, x_{1}\right)=\int_{-\infty}^{\infty} d t e^{i \omega t}\left\langle 0\left|\left\{\rho_{a}\left(t, x_{1}\right), \rho_{b}\left(0, x_{2}\right)\right\}\right| 0\right\rangle \\
& =\frac{\nu}{2 \pi}|\omega| \delta_{a, b} e^{i \omega a\left(x_{1}-x_{2}\right)}
\end{aligned}
$$

so that, in particular, $S_{a a}^{(0)}\left(\omega ; x_{1}, x_{1}\right)=\frac{\nu}{2 \pi}|\omega|$.

Combining the zeroth and second order results, we obtain the results used in section III for the noise in incoming $\left(a x_{1}<0\right)$ and outgoing $\left(a x_{1}>0\right)$ branches, namely:

$$
S(\omega)= \begin{cases}\frac{\nu}{2 \pi}|\omega|, & \text { incoming branches } \\ \frac{\nu}{2 \pi}|\omega|+\frac{4 \pi \nu g}{\Gamma(2 g)}|\Gamma|^{2}|| \omega|-| \omega_{0}||^{2 g-1} \theta\left(\left|\omega_{0}\right|-|\omega|\right), & \text { outgoing branches }\end{cases}
$$


It is straightforward to show that the noise in the incoming branch remains equal to $\frac{\nu}{2 \pi}|\omega|$ to all orders in perturbation theory.

Next, we will obtain correlations between densities of an incoming and an outgoing branch (the cross-correlations). Without loss of generatity, we will focus on the correlations between two $R$ branches $(a=1)$, one outgoing $\left(x_{1}>0\right)$, and another incoming $\left(x_{2}<0\right)$. The results for other combinations of branches are trivially obtained from the case we consider. We have, again, all the tools at hand, namely Eqs. (A13) and (A14), as well as our expressions for $g_{\mu \nu}^{a b}(\omega, x)$ and $\tilde{P}_{\mu \nu}(\omega)$. We find

$$
\begin{aligned}
S_{R R}^{(2)}\left(\omega ; x_{1}>0, x_{2}<0\right) & =\int_{-\infty}^{\infty} d t e^{i \omega t}\left\langle\left\{\rho_{R}\left(t, x_{1}>0\right), \rho_{R}\left(0, x_{2}<0\right)\right\}\right\rangle_{|\Gamma|^{2}} \\
& =e^{i \omega\left(x_{1}-x_{2}\right)} \frac{|\Gamma|^{2} g \nu}{2}\left\{\operatorname{sign}(\omega) H_{g}(\omega)\right. \\
& \left.-\frac{2 \pi}{\Gamma(2 g)}\left[\left(|\omega|+\left|\omega_{0}\right|\right)^{2 g-1}+|| \omega|-| \omega_{0}||^{2 g-1} \operatorname{sign}\left(|\omega|-\left|\omega_{0}\right|\right)\right]\right\},
\end{aligned}
$$

where the function $H_{g}(\omega)$ is defined as

$$
\begin{aligned}
H_{g}(\omega) & =2\left[t\left(\omega_{0}\right)-b\left(\omega_{0}\right)\right]-\left[t\left(\omega_{0}-\omega\right)-b\left(\omega_{0}-\omega\right)\right]-\left[t\left(\omega_{0}+\omega\right)-b\left(\omega_{0}+\omega\right)\right] \\
& =8 \int_{0}^{\infty} d t \cos \left(\omega_{0} t\right) \sin ^{2}(\omega t / 2)\left[\frac{1}{(\delta+i t)^{2 g}}-\frac{1}{(\delta-i t)^{2 g}}\right] .
\end{aligned}
$$

One can show particularly that $H_{1 / 2}(\omega)=-4 i \ln \left(\left|\frac{\omega^{2}-\omega_{0}^{2}}{\omega_{0}^{2}}\right|\right), H_{1}(\omega)=0$, and $H_{2}(\omega)=-\frac{4 i \omega^{2}}{3 \delta} \rightarrow \infty$ as $\delta \rightarrow 0$.

The zero order contribution to the cross-correlations is read directly from Eq. (A18): $S_{R R}^{(0)}\left(\omega ; x_{1}>0, x_{2}<0\right)=$ $\frac{\nu}{2 \pi}|\omega| e^{i \omega\left(x_{1}-x_{2}\right)}$.

\section{APPENDIX B: PERTURBATIVE CALCULATION FOR THE DENSITY-DENSITY COUPLING}

Here we consider the neutral coupling $L_{\text {int }}=\gamma\left(\rho_{R}(t, 0)-\rho_{L}(t, 0)\right)^{2}$, and show that it contributes to the correlations between incoming and outgoing branches, although it does not contribute to correlations between two incoming or two outgoing ones. The calculations are simpler than the ones in Appendix A. We will demonstrate the point by calculating the correlation $\left\langle T_{c}\left(\rho_{R}\left(t, x_{1}\right) \rho_{R}\left(0, x_{2}\right)\right)\right\rangle$ to first and second order in $\gamma$. Other correlations can be calculated in a very similar way.

As in Appendix A, contour integrals are simplified by keeping track of insertions in the top and bottom branches with indices $\mu, \nu= \pm 1$. It is useful to define

$$
\begin{aligned}
h_{\mu \nu}^{a b}\left(t_{1}, x_{1} ; t_{2}, x_{2}\right) & =h_{\mu \nu}^{a b}\left(t_{1}-t_{2}, x_{1}-x_{2}\right)=\left\langle 0\left|T_{c}\left(\rho_{a}\left(t_{1}^{\mu}, x_{1}\right) \rho_{b}\left(t_{2}^{\nu}, x_{2}\right)\right)\right| 0\right\rangle \\
& =\frac{\nu}{(2 \pi)^{2}} \partial_{x_{1}} \partial_{x_{2}}\left\langle 0\left|T_{c}\left(\phi_{a}\left(t_{1}^{\mu}, x_{1}\right) \phi_{b}\left(t_{2}^{\nu}, x_{2}\right)\right)\right| 0\right\rangle \\
& =\frac{\nu}{(2 \pi)^{2}} \partial_{x_{1}} \partial_{x_{2}} G_{\mu \nu}^{a b}\left(t_{1}-t_{2}, x_{1}-x_{2}\right),
\end{aligned}
$$

where $a$ and $b$, as in Appendix A, take the values +1 for $R$ moving branches and -1 for $L$ moving ones. It follows from the calculations of Appendix A that $\tilde{h}_{\mu \nu}^{a b}(\omega, x)=-\frac{\nu}{(2 \pi)^{2}} \partial_{x}^{2} \tilde{G}_{\mu \nu}^{a b}(\omega, x)=-\frac{\nu}{(2 \pi)^{2}} \partial_{x} g_{\mu \nu}^{a b}(\omega, x)$, which gives

$$
\tilde{h}_{\mu \nu}^{a b}(\omega, x)=\frac{\nu}{(2 \pi)^{2}} \delta_{a, b} \times \begin{cases}2 \pi|\omega| \theta(a \omega x) e^{i \omega a x}-2 \pi i \delta(x), & \mu=+1, \nu=+1 \\ 2 \pi|\omega| \theta(-a \omega x) e^{i \omega a x}+2 \pi i \delta(x), & \mu=-1, \nu=-1 \\ 2 \pi|\omega| \theta(-\omega) e^{i \omega a x}, & \mu=+1, \nu=-1 \\ 2 \pi|\omega| \theta(\omega) e^{i \omega a x}, & \mu=-1, \nu=+1\end{cases}
$$

The perturbative results can be easily written in terms of these $\tilde{h}$ 's. 
Notice that the only term in the interaction $\rho_{-}^{2}=\rho_{R}^{2}-2 \rho_{R} \rho_{L}+\rho_{L}^{2}$ that contributes to $\left\langle T_{c}\left(\rho_{R}\left(t, x_{1}\right) \rho_{R}\left(0, x_{2}\right)\right)\right\rangle$ to order $\gamma$ is the $\rho_{R}^{2}$ term. The first order in $\gamma$ correction to the correlation function can be written as

$$
\begin{aligned}
& \left\langle T_{c}\left(\rho_{R}\left(t, x_{1}\right) \rho_{R}\left(0, x_{2}\right)\right)\right\rangle_{\gamma} \\
& =i \gamma \oint_{c} d t_{1}\left\langle 0\left|T_{c}\left(\rho_{R}\left(t, x_{1}\right) \rho_{R}\left(0, x_{2}\right) \rho_{R}\left(t_{1}, 0\right) \rho_{R}\left(t_{1}, 0\right)\right)\right| 0\right\rangle \\
& =2 i \gamma \oint_{c} d t_{1}\left\langle 0\left|T_{c}\left(\rho_{R}\left(t, x_{1}\right) \rho_{R}\left(t_{1}, 0\right)\right)\right| 0\right\rangle \times\left\langle 0\left|T_{c}\left(\rho_{R}\left(0, x_{2}\right) \rho_{R}\left(t_{1}, 0\right)\right)\right| 0\right\rangle .
\end{aligned}
$$

The Fourier transform $F_{R R}^{(1)}\left(\omega ; x_{1}, x_{2}\right)$ of the expression in Eq. B33) is simply

$$
F_{R R}^{(1)}\left(\omega ; x_{1}, x_{2}\right)=2 i \gamma \sum_{\mu} \operatorname{sgn}(\mu) \tilde{h}_{+\mu}^{++}\left(\omega, x_{1}\right) \tilde{h}_{+\mu}^{++}\left(-\omega, x_{2}\right)
$$

and thus to first order in $\gamma$ the cross-correlation spectrum is given by

$$
\begin{aligned}
S_{R R}^{(1)}\left(\omega ; x_{1}, x_{2}\right) & =S_{R R}^{(1)}\left(-\omega ; x_{2}, x_{1}\right)=\int_{-\infty}^{\infty} d t e^{i \omega t}\left\langle\left\{\rho_{R}\left(t, x_{1}\right), \rho_{R}\left(0, x_{2}\right)\right\}\right\rangle_{\gamma} \\
& =F_{R R}^{(1)}\left(\omega ; x_{1}, x_{2}\right)+F_{R R}^{(1)^{*}}\left(-\omega ; x_{1}, x_{2}\right) \\
& =\frac{i \gamma \nu^{2}}{2 \pi^{2}} \theta\left(-x_{1} x_{2}\right) \omega^{2} \operatorname{sign}\left(\omega x_{1}\right) e^{i \omega\left(x_{1}-x_{2}\right)} .
\end{aligned}
$$

Turning now to second order in the perturbation expansion, both the $\rho_{R}^{2}$ and the $\rho_{R} \rho_{L}$ terms in the interaction $\rho_{-}^{2}=\rho_{R}^{2}-2 \rho_{R} \rho_{L}+\rho_{L}^{2}$ can contribute to the order $\gamma^{2}$ correction to $\left\langle T_{c}\left(\rho_{R}\left(t, x_{1}\right) \rho_{R}\left(0, x_{2}\right)\right)\right\rangle$. Consider the $\gamma \rho_{R}^{2}$ coupling, so that to second order we have

$$
\begin{aligned}
& \left\langle T_{c}\left(\rho_{R}\left(t, x_{1}\right) \rho_{R}\left(0, x_{2}\right)\right)\right\rangle\left(\gamma \rho_{R}^{2}\right)^{2} \\
& =\frac{(i \gamma)^{2}}{2 !} \oint_{c} d t_{1} \oint_{c} d t_{2}\langle 0| \\
& \left.=8 \frac{(i \gamma)^{2}}{2 !} \oint_{c} d \rho_{R} \oint_{c} d t_{2} \quad\left\langle 0\left|T_{c}\left(\rho_{R}\left(t, x_{1}\right) \rho_{R}\left(0, x_{2}\right) \rho_{R}\left(t_{1}, 0\right)\right)\right| 0\right\rangle \rho_{R}\left(t_{1}, 0\right) \rho_{R}\left(t_{2}, 0\right) \rho_{R}\left(t_{2}, 0\right)\right)|0\rangle \\
& \times\left\langle 0\left|T_{c}\left(\rho_{R}\left(0, x_{2}\right) \rho_{R}\left(t_{2}, 0\right)\right)\right| 0\right\rangle \\
& \times\left\langle 0\left|T_{c}\left(\rho_{R}\left(t_{1}, 0\right) \rho_{R}\left(t_{2}, 0\right)\right)\right| 0\right\rangle .
\end{aligned}
$$

The effect of the $\gamma 2 \rho_{R} \rho_{L}$ coupling can be calculated likewise. The Fourier transform of these two contributions combined give

$$
F_{R R}^{(2)}\left(\omega ; x_{1}, x_{2}\right)=-4 \gamma^{2} \sum_{\mu \nu} \operatorname{sign}(\mu \nu) \tilde{h}_{+\mu}^{++}\left(\omega, x_{1}\right) \tilde{h}_{+\nu}^{++}\left(-\omega, x_{2}\right)\left[\tilde{h}_{\mu \nu}^{++}(\omega, 0)+\tilde{h}_{\mu \nu}^{--}(\omega, 0)\right],
$$

so that the cross-correlation spectrum to second order is

$$
\begin{aligned}
S_{R R}^{(2)}\left(\omega ; x_{1}, x_{2}\right) & =S_{R R}^{(2)}\left(-\omega ; x_{2}, x_{1}\right)=\int_{-\infty}^{\infty} d t e^{i \omega t}\left\langle\left\{\rho_{R}\left(t, x_{1}\right), \rho_{R}\left(0, x_{2}\right)\right\}\right\rangle_{\gamma^{2}} \\
& =F_{R R}^{(2)}\left(\omega ; x_{1}, x_{2}\right)+F_{R R}^{(2)^{*}}\left(-\omega ; x_{1}, x_{2}\right) \\
& =-\frac{\gamma^{2} \nu^{3}}{2 \pi^{3}} \theta\left(-x_{1} x_{2}\right) e^{i \omega\left(x_{1}-x_{2}\right)}\left[|\omega|^{3}-2 i " \delta(0)^{\prime \prime} \omega^{2} \operatorname{sign}\left(\omega x_{1}\right)\right],
\end{aligned}
$$

where " $\delta(0)$ " is a regulation dependent divergent term.

Notice that, both to first and second order in $\gamma$, the correlations on the same side of the junction, i.e., $x_{1} x_{2}>0$, do not feel the density-density coupling, whereas correlations across the junction $\left(x_{1} x_{2}<0\right)$ do feel the coupling. More generally, when one considers all possible correlations involving $R$ and $L$ branches, only those which contain an incoming and an outgoing branch will have a non-zero correction due to the density-density coupling. Correlations between two incoming or two outgoing branches will be zero. 


\section{APPENDIX C: SCATTERING CALCULATION}

In this appendix, we evaluate the expectation values and integrals used for calculating the noise in Section IV. The methods of calculation are very similar to those in [18]

First, we will evaluate the noise in the incoming reservoir, which is given by

$$
S\left(\omega ; x_{-}, x_{-}\right)=\int_{-\infty}^{\infty} d t\left(e^{i \omega t}+e^{-i \omega t}\right)\left\langle\rho_{-}\left(t, x_{-}\right) \rho_{-}\left(0, x_{-}\right)\right\rangle .
$$

The expectation value we must calculate is given by

$$
\left\langle\rho_{-}\left(t, x_{-}\right) \rho_{-}\left(0, x_{-}\right)\right\rangle=\left\langle\psi^{\dagger}\left(t, x_{-}\right) \psi\left(t, x_{-}\right) \psi^{\dagger}\left(0, x_{-}\right) \psi\left(0, x_{-}\right)\right\rangle \text {, }
$$

with $x_{-}<0$. Using the solutions in Eqs. (22) and 23 for $\psi$ and $\psi^{\dagger}$, we find

$$
\left\langle\rho_{-}\left(t, x_{-}\right) \rho_{-}\left(0, x_{-}\right)\right\rangle=\sum_{\omega_{1}, \omega_{2}, \omega_{3}, \omega_{4}} e^{-i\left(\omega_{1}+\omega_{2}\right) t}\left\langle\Phi\left|A_{-\omega_{1}}^{\dagger} A_{\omega_{2}} A_{-\omega_{3}}^{\dagger} A_{\omega_{4}}\right| \Phi\right\rangle e^{i\left(\omega_{1}+\omega_{2}+\omega_{3}+\omega_{4}\right) x_{-}} .
$$

The connected part of $\left\langle A_{-\omega_{1}}^{\dagger} A_{\omega_{2}} A_{-\omega_{3}}^{\dagger} A_{\omega_{4}}\right\rangle$ has $A_{-\omega_{1}}^{\dagger}$ paired with $A_{\omega_{4}}$ and $A_{\omega_{2}}$ paired with $A_{-\omega_{3}}^{\dagger}$ and is given by

$$
\left\langle\Phi\left|A_{-\omega_{1}}^{\dagger} A_{\omega_{2}} A_{-\omega_{3}}^{\dagger} A_{\omega_{4}}\right| \Phi\right\rangle_{\mathrm{con}}=\left\langle\Phi\left|A_{-\omega_{1}}^{\dagger} A_{\omega_{4}}\right| \Phi\right\rangle\left\langle\Phi\left|A_{\omega_{2}} A_{-\omega_{3}}^{\dagger}\right| \Phi\right\rangle
$$

Evaluating these correlations using equations (26) and 30, we find that the current-current correlation reduces to

$$
\left\langle\rho_{-}\left(t, x_{-}\right) \rho_{-}\left(0, x_{-}\right)\right\rangle=\sum_{\omega_{1}, \omega_{2}} e^{-i\left(\omega_{1}+\omega_{2}\right) t} n_{-\omega_{1}}\left(1-n_{\omega_{2}}\right) .
$$

Substituting this expression back into equation (34) for the noise, and performing the integrals over $t$ and $\omega_{1}$, we obtain

$$
S\left(\omega ; x_{-}, x_{-}\right)(\omega)=\int_{-\infty}^{\infty} \frac{d \omega_{2}}{2 \pi} n_{\omega_{2}-\omega}\left(1-n_{\omega_{2}}\right)+\int_{-\infty}^{\infty} \frac{d \omega_{2}}{2 \pi} n_{\omega_{2}+\omega}\left(1-n_{\omega_{2}}\right) .
$$

At zero temperature, the integrands are given by

$$
n_{\omega_{2} \mp \omega}\left(1-n_{\omega_{2}}\right)= \begin{cases}1 & \text { for } \pm \omega>0 \text { and } \omega_{0} \leq \omega_{2} \leq \omega_{0} \pm \omega \\ 0 & \text { otherwise }\end{cases}
$$

Performing the integral, we obtain the desired result:

$$
S\left(\omega ; x_{-}, x_{-}\right)(\omega)=\frac{1}{2 \pi}|\omega|
$$

Next, we will calculate the noise in the outgoing current, which is given by

$$
S\left(\omega ; x_{+}, x_{+}\right)=\int_{-\infty}^{\infty} d t\left(e^{i \omega t}+e^{-i \omega t}\right)\left\langle\rho_{-}\left(t, x_{+}\right) \rho_{-}\left(0, x_{+}\right)\right\rangle
$$

where $x_{+}>0$. This time we must evaluate the expectation value

$$
\left\langle\rho_{-}\left(t, x_{+}\right) \rho_{-}\left(0, x_{+}\right)\right\rangle=\left\langle\psi^{\dagger}\left(t, x_{+}\right) \psi\left(t, x_{+}\right) \psi^{\dagger}\left(0, x_{+}\right) \psi\left(0, x_{+}\right)\right\rangle,
$$

According to equations (22) and (23), this is equal to

$$
\left\langle\rho_{-}\left(t, x_{+}\right) \rho_{-}\left(0, x_{+}\right)\right\rangle=\sum_{\omega_{1}, \omega_{2}, \omega_{3}, \omega_{4}} e^{-i\left(\omega_{1}+\omega_{2}\right) t}\left\langle\Phi\left|B_{-\omega_{1}}^{\dagger} B_{\omega_{2}} B_{-\omega_{3}}^{\dagger} B_{\omega_{4}}\right| \Phi\right\rangle e^{i\left(\omega_{1}+\omega_{2}+\omega_{3}+\omega_{4}\right) x_{+}} .
$$

Because the scattering states are defined in terms of the operator $A_{\omega}$, we will use equation (24) to rewrite all the $B$ 's in terms of the $A$ 's, with the result

$$
\left\langle B_{-\omega_{1}}^{\dagger} B_{\omega_{2}} B_{-\omega_{3}}^{\dagger} B_{\omega_{4}}\right\rangle=s_{o}+s_{t}
$$


In this equation, $s_{o}$ describes events where at both time 0 and at time $t$ one particle is destroyed and another is created. The second term, $s_{t}$, describes events where at one time two particles are created, and at the other time two are destroyed. All the other terms in the correlation function of the four $B$ 's will vanish. $s_{o}$ and $s_{t}$ are given by

$$
\begin{aligned}
s_{O}=\frac{1}{16} & {\left[c_{\omega_{1}} c_{\omega_{2}} c_{\omega_{3}} c_{\omega_{4}}\left\langle A_{-\omega_{1}}^{\dagger} A_{\omega_{2}} A_{-\omega_{3}}^{\dagger} A_{\omega_{4}}\right\rangle\right.} \\
& +c_{\omega_{1}} c_{\omega_{2}} d_{\omega_{3}} d_{\omega_{4}}\left\langle A_{-\omega_{1}}^{\dagger} A_{\omega_{2}} A_{\omega_{3}} A_{-\omega_{4}}^{\dagger}\right\rangle \\
& +d_{\omega_{1}} d_{\omega_{2}} c_{\omega_{3}} c_{\omega_{4}}\left\langle A_{\omega_{1}} A_{-\omega_{2}}^{\dagger} A_{-\omega_{3}}^{\dagger} A_{\omega_{4}}\right\rangle \\
& \left.+d_{\omega_{1}} d_{\omega_{2}} d_{\omega_{3}} d_{\omega_{4}}\left\langle A_{\omega_{1}} A_{-\omega_{2}}^{\dagger} A_{\omega_{3}} A_{-\omega_{4}}^{\dagger}\right\rangle\right]
\end{aligned}
$$

and

$$
\begin{aligned}
s_{t}=\frac{1}{16} & {\left[d_{\omega_{1}} c_{\omega_{2}} c_{\omega_{3}} d_{\omega_{4}}\left\langle A_{\omega_{1}} A_{\omega_{2}} A_{-\omega_{3}}^{\dagger} A_{-\omega_{4}}^{\dagger}\right\rangle\right.} \\
& \left.+c_{\omega_{1}} d_{\omega_{2}} d_{\omega_{3}} c_{\omega_{4}}\left\langle A_{-\omega_{1}}^{\dagger} A_{-\omega_{2}}^{\dagger} A_{\omega_{3}} A_{\omega_{4}}\right\rangle\right] .
\end{aligned}
$$

In these equations, $c_{\omega}$ and $d_{\omega}$ are given by

$$
c_{\omega}=1+e^{i \phi(\omega)} \quad \text { and } \quad d_{\omega}=1-e^{i \phi(\omega)},
$$

with $\phi(\omega)$ defined in Eq. (25). The correlations of the four $A$ 's can be evaluated using Eqs. (26) and (30). If we interchange $\omega_{1}$ with $\omega_{2}$ in the second two lines of $s_{o}$, and perform the sums over $\omega_{3}$ and $\omega_{4}$, we obtain

$$
\begin{aligned}
\sum_{\omega_{3}, \omega_{4}} s_{O} e^{i\left(\omega_{1}+\omega_{2}+\omega_{3}+\omega_{4}\right) x_{+}=\frac{1}{16}} & {\left[c_{\omega_{1}} c_{\omega_{2}} c_{-\omega_{1}} c_{-\omega_{2}}-c_{\omega_{1}} c_{\omega_{2}} d_{-\omega_{1}} d_{-\omega_{2}}\right.} \\
& \left.-d_{\omega_{1}} d_{\omega_{2}} c_{-\omega_{1}} c_{-\omega_{2}}+d_{\omega_{1}} d_{\omega_{2}} d_{-\omega_{1}} d_{-\omega_{2}}\right] \\
& \times n_{-\omega_{1}}\left(1-n_{\omega_{2}}\right) .
\end{aligned}
$$

In this equation, the expression containing the number operators is the same as for the noise in the incoming current, so we will obtain the same limits of integration as in equation (C7). Next, we can expand out the $c_{\omega}$ 's and $d_{\omega}$ 's in terms of $\omega$ and substitute this back into equation (34) for the noise. After performing the integrals over $t$ and $\omega_{1}$, we find that the contribution to the noise due to $s_{o}$ has the form

$$
S_{o}\left(\omega ; x_{+}, x_{+}\right)=\int_{\omega_{0}}^{\omega_{0} \pm \omega} \frac{d \omega_{2}}{2 \pi} \frac{\left(\left(4 \pi|\Gamma|^{2}\right)^{2}-\omega_{2}\left(\omega_{2} \mp \omega_{1}\right)\right)^{2}}{\left(\left(\omega_{2} \mp \omega\right)^{2}+\left(4 \pi|\Gamma|^{2}\right)^{2}\right)\left(\omega_{2}^{2}+\left(4 \pi|\Gamma|^{2}\right)^{2}\right)} \theta( \pm \omega),
$$

where we sum over the two different signs in front of $\omega$. Upon performing the $\omega_{2}$ integral, we obtain

$$
\begin{aligned}
S_{o}\left(\omega ; x_{+}, x_{+}\right)=\frac{|\omega|}{2 \pi}-2|\Gamma|^{2}[ & \left.\tan ^{-1}\left(\frac{|\omega|-\omega_{0}}{4 \pi|\Gamma|^{2}}\right)+\tan ^{-1}\left(\frac{|\omega|+\omega_{0}}{4 \pi|\Gamma|^{2}}\right)\right] \\
-8 \pi \frac{|\Gamma|^{4}}{|\omega|} & {\left[2 \ln \left(\omega_{0}^{2}+\left(4 \pi|\Gamma|^{2}\right)^{2}\right)-\ln \left(\left(\omega+\omega_{0}\right)^{2}+\left(4 \pi|\Gamma|^{2}\right)^{2}\right)\right.} \\
& \left.-\ln \left(\left(\omega-\omega_{0}\right)^{2}+\left(4 \pi|\Gamma|^{2}\right)^{2}\right)\right] .
\end{aligned}
$$

Next, we will calculate the contribution to the noise due to $s_{t}$. The two expectation values we must evaluate are $\left\langle A_{\omega_{1}} A_{\omega_{2}} A_{-\omega_{3}}^{\dagger} A_{-\omega_{4}}^{\dagger}\right\rangle$ and $\left\langle A_{-\omega_{1}}^{\dagger} A_{-\omega_{2}}^{\dagger} A_{\omega_{3}} A_{\omega_{4}}\right\rangle$. In both cases, either $\omega_{1}$ is paired with $\omega_{3}$ and $\omega_{2}$ is paired with $\omega_{4}$, or $\omega_{1}$ is paired with $\omega_{4}$ and $\omega_{2}$ with $\omega_{3}$. Thus we have

$$
\left\langle A_{\omega_{1}} A_{\omega_{2}} A_{-\omega_{3}}^{\dagger} A_{-\omega_{4}}^{\dagger}\right\rangle=\left(1-n_{\omega_{1}}\right)\left(1-n_{\omega_{2}}\right)\left(\delta_{\omega_{1},-\omega_{4}} \delta_{\omega_{2},-\omega_{3}}-\delta_{\omega_{1},-\omega_{3}} \delta_{\omega_{2},-\omega_{4}}\right)
$$

and 


$$
\left\langle A_{-\omega_{1}}^{\dagger} A_{-\omega_{2}}^{\dagger} A_{\omega_{3}} A_{\omega_{4}}\right\rangle=n_{-\omega_{1}} n_{-\omega_{2}}\left(\delta_{-\omega_{1}, \omega_{4}} \delta_{-\omega_{2}, \omega_{3}}-\delta_{-\omega_{1}, \omega_{3}} \delta_{-\omega_{2}, \omega_{4}}\right) .
$$

Substituting these expressions into the equation for $s_{t}$ and performing the integrals over $\omega_{3}$ and $\omega_{4}$, we obtain

$$
\begin{aligned}
\sum_{\omega_{3}, \omega_{4}} s_{t} e^{i\left(\omega_{1}+\omega_{2}+\omega_{3}+\omega_{4}\right) x_{+}}=\frac{1}{16} & {\left[d_{\omega_{1}} d_{-\omega_{1}} c_{\omega_{2}} c_{-\omega_{2}}-d_{\omega_{1}} c_{-\omega_{1}} c_{\omega_{2}} d_{-\omega_{2}}\right] } \\
\times & {\left[\left(1-n_{\omega_{1}}\right)\left(1-n_{\omega_{2}}\right)+n_{-\omega_{1}} n_{-\omega_{2}}\right] . }
\end{aligned}
$$

When we expand the $c$ 's and $d$ 's in terms of $\omega$ and perform the integral over $t$, we find that the contribution to the noise due to $s_{t}$ is given by

$$
\begin{aligned}
S_{t}\left(\omega ; x_{+}, x_{+}\right)=\int \frac{d \omega_{1}}{2 \pi} \frac{d \omega_{2}}{2 \pi} \frac{\left(4 \pi|\Gamma|^{2}\right)^{2}\left(\omega_{2}^{2}-\omega_{1} \omega_{2}\right)}{\left(\omega_{1}^{2}+\left(4 \pi|\Gamma|^{2}\right)^{2}\right)\left(\omega_{2}^{2}+\left(4 \pi|\Gamma|^{2}\right)^{2}\right)} \\
\\
\quad \times \delta\left(\omega_{1}+\omega_{2} \pm \omega\right)\left[\left(1-n_{\omega_{1}}\right)\left(1-n_{\omega_{2}}\right)+n_{-\omega_{1}} n_{-\omega_{2}}\right]
\end{aligned}
$$

where again it is understood that we sum the two integrands with the different sign in front of $\omega$. After the integration over $\omega_{1}$ is performed, the expression in square brackets becomes

$$
\left(1-n_{-\omega_{2} \mp \omega}\right)\left(1-n_{\omega_{2}}\right)+n_{\omega_{2} \pm \omega} n_{-\omega_{2}}= \begin{cases}1 & \text { for } \omega_{0}<\omega_{2}<\mp \omega-\omega_{0} \text { and } \mp \omega-2 \omega_{0}>0 \\ 1 & \text { for }-\omega_{0}<\omega_{2}<\mp \omega+\omega_{0} \text { and } \mp \omega+2 \omega_{0}>0 \\ 0 & \text { otherwise. }\end{cases}
$$

We note that this time the limits of integration determined by the factors of $n$ impose cutoffs at $\omega= \pm 2 \omega_{0}$. These are the origins of the singularities at $\omega=2 \omega_{0}$, which, as we shall see shortly, persist for all $|\Gamma| \neq 0$. After equation (C23) is substituted into the equation for $S_{t}\left(\omega ; x_{+}, x_{+}\right)$, the noise becomes

$$
S_{t}\left(\omega ; x_{+}, x_{+}\right)=\sum_{a, b= \pm 1} \theta\left(a \omega+b 2 \omega_{0}\right) \int_{-b \omega_{0}}^{b \omega_{0}-a \omega} \frac{d \omega_{2}}{2 \pi} \frac{\left(4 \pi|\Gamma|^{2}\right)^{2}\left(\omega_{2}^{2}+\omega_{2}\left(\omega_{2}+a \omega\right)\right)}{\left(\left(\omega_{2}+a \omega\right)^{2}+\left(4 \pi|\Gamma|^{2}\right)^{2}\right)\left(\omega_{2}^{2}+\left(4 \pi|\Gamma|^{2}\right)^{2}\right)} .
$$

The integration over $\omega_{2}$ yields

$$
\begin{aligned}
S_{t}\left(\omega ; x_{+}, x_{+}\right)=\sum_{a, b= \pm 1} \theta & \left(a \omega+b 2 \omega_{0}\right)\left\{2|\Gamma|^{2}\left[\tan ^{-1}\left(\frac{b \omega_{0}}{4 \pi|\Gamma|^{2}}\right)+\tan ^{-1}\left(\frac{a \omega+b \omega_{0}}{4 \pi|\Gamma|^{2}}\right)\right]\right. \\
+ & \left.\frac{8 \pi|\Gamma|^{4}}{a \omega}\left[\ln \left(\left(4 \pi|\Gamma|^{2}\right)^{2}+\omega_{0}^{2}\right)-\ln \left(\left(4 \pi|\Gamma|^{2}\right)^{2}+\left(a \omega+b \omega_{0}\right)^{2}\right)\right]\right\} .
\end{aligned}
$$

We note that this contribution to the noise has the step function which provides a "sharp" singularity at $|\omega|=\left|2 \omega_{0}\right|$, for any non-zero value of $|\Gamma|$. This is the electron singularity. However, for $|\Gamma| \rightarrow 0$, the arctangents provide a singularity at $|\omega|=\left|\omega_{0}\right|$, which is the quasiparticle singularity.

When we add the two contributions to the noise together, we find that the noise on the outgoing side of the impurity is

$$
\begin{aligned}
S\left(\omega ; x_{+}, x_{+}\right)=\frac{|\omega|}{2 \pi}+ & \theta\left(2\left|\omega_{0}\right|-|\omega|\right)\left\{4|\Gamma|^{2}\left[\tan ^{-1}\left(\frac{\left|\omega_{0}\right|}{4 \pi|\Gamma|^{2}}\right)+\tan ^{-1}\left(\frac{\left|\omega_{0}\right|-|\omega|}{4 \pi|\Gamma|^{2}}\right)\right]\right. \\
& \left.+16 \pi \frac{|\Gamma|^{4}}{|\omega|}\left[\ln \left(\left(4 \pi|\Gamma|^{2}\right)^{2}+\left(|\omega|-\left|\omega_{0}\right|\right)^{2}\right)-\ln \left(\left(4 \pi|\Gamma|^{2}\right)^{2}+\omega_{0}^{2}\right)\right]\right\} .
\end{aligned}
$$

Finally, the noise between the currents on either side of the impurity can be calculated similarly, so we will omit the details here. 
[1] X. G. Wen, Phys. Rev. B 41, 12838 (1990).

[2] X. G. Wen, Phys. Rev. B 44, 5708 (1991).

[3] C. L. Kane and Matthew P. A. Fisher, Phys. Rev. Lett. 68, 1220 (1992); Phys. Rev. B 46, 15233 (1992); Phys. Rev. Lett. 72, 724 (1994).

[4] Akira Furusaki and Naoto Nagaosa, Phys. Rev. B 47, 3827 (1993); Phys. Rev. B 47, 4631 (1993).

[5] C. de C. Chamon and X. G. Wen, Phys. Rev. Lett. 70, 2605 (1993).

[6] F. P. Milliken, C. P. Umbach and R. A. Webb, preprint.

[7] K. Moon, H. Yi, C. L. Kane, S. M. Girvin, and Matthew P. A. Fisher, Phys. Rev. Lett. 71, 4381 (1993).

[8] F. Guinea, G. Gomez-Santos, M. Sasseti, and U. Ueda, cond-mat/9411130.

[9] C. H. Mak, Reinhold Egger, Maura Sasseti, and Ulrich Weiss, preprint.

[10] R. Egger, M. Sasseti and U. Weiss, cond-mat/9504040.

[11] P. Fendley, A. W. W. Ludwig, and H. Saleur, Phys. Rev. Lett. 74, 3005 (1995).

[12] P. Fendley, A. W. W. Ludwig, and H. Saleur, cond-mat/9503172.

[13] A. Schmid, Phys. Rev. Lett. 51 (1983) 1506; M. P. A. Fisher and W. Zwerger, Phys. Rev. B 32 (1985) 6190; C. G. Callan and D. E. Freed, Nucl. Phys. B 374 (1992), 543.

[14] C. L. Kane and Matthew P. A. Fisher, Phys. Rev. Lett. 72, 724 (1994).

[15] C. de C. Chamon, D. E. Freed, and X. G. Wen, Phys. Rev. B 51, 2363 (1995).

[16] P. Fendley, A. W. W. Ludwig, and H. Saleur, cond-mat/9505031.

[17] Rolf Landauer, Phys. Rev. B 47, 16427 (1993) and references therein.

[18] M. Buttiker, Phys. Rev. B 46, 12485 (1992) and references therein.

[19] G. B. Lesovik, Pis'ma Zh. Eksp. Teor. Fiz. 49, 513 (1989) [JETP LETT. 49, 592 (1989)].

[20] Xiao-Gang Wen, Intl. J. of Mod. Phys. B 6, 1711 (1992).

[21] S.-R. Eric Yang, Solid State Comm. 81, 375 (1992).

[22] F. Guinea, Phys. Rev. B 32, 7518 (1985).

[23] K. A. Matveev, Phys. Rev. B 51, 1743 (1995); A. Furusaki and K. A. Matveev, cond-mat/9505035.

[24] R. Floreanini and R. Jackiw, Phys. Rev. Lett. 59, 1873 (1987).

[25] M. B. Green, J. H. Schwartz and E. Witten, Superstring theory, volume I, Cambridge University Press, Cambridge, 1988. 


\section{FIGURE CAPTIONS}

Figure 1. Geometries for tunneling between edge states. By adjusting the gate voltage $V_{G}$ one can obtain either a simply connected QH droplet (a), or two disconnected QH droplets (b). For the geometry in (a) both electrons and quasiparticles (carrying fractional charge) can tunnel from one edge to the other, whereas for the tunneling geometry in (b) only electrons can tunnel. The Luttinger liquid behavior is characterized by the exponent $g=\nu$ in (a), and $g=\nu^{-1}$ in (b). The tunneling current $I_{t}$ depends on the applied voltage between the right and left edges, and by increasing this voltage one can also cross over from the geometry (b) to the geometry (a).

Figure 2. Four terminal geometry for the measurement of tunneling between edge states. The terminals 1 and 2 correspond to branches that are incoming to the scatterer, while terminals 3 and 4 correspond to outgoing ones. The arrows indicate the direction of propagation for a given branch. The incoming branches are in equilibrium with their reservoirs of origin, while the outgoing ones do get effected by the scatterer. By measuring fluctuations in the voltages/currents at the four terminals $\left(V_{i} / I_{i}, i=1,2,3,4\right)$, the auto-correlation spectra $S_{i j}(\omega)$, with $i=j$, and the cross-correlation spectra $S_{i j}(\omega)$, with $i \neq j$, can be obtained. These voltage/current fluctuations contain information on the fluctuations of the tunneling current.

Figure 3. Plots of the excess noise of outgoing branches (probes 3 and 4 of Fig. 2) calculated to second order in perturbation theory (equation (11)). The excess noise is normalized to the zero- frequency shot noise level, and the frequency $\omega$ to the Josephson frequency $\omega_{J}\left(S^{(2)}(\omega) / 2 e^{*} I_{t}\right.$ vs. $\left.\omega / \omega_{J}\right)$. Different singularities are obtained at $\omega=\omega_{J}$ for different values of $g: \frac{1}{3}, \frac{1}{2}, \frac{2}{3}, 1$, and 2 . One should keep in mind that, although the singularities all occur at $\omega=\omega_{J}$, the value of $\omega_{J}$ depends on the charge of the current carrier, which in turn also depends on $g$.

Figure 4. A particle (plane wave) incoming from the left $(x<0)$ with energy $\omega$ scatters off the impurity at $x=0$ into a superposition of a particle at energy $\omega$ and a hole at energy $-\omega$ on the right side of the impurity $(x>0)$. In the case where the incoming state is a filled Fermi sea up to the energy $\omega_{0}$, the scattered state on the right side of the impurity will be completely filled up to energy $-\omega_{0}$, and partially filled between $-\omega_{0}$ and $\omega_{0}$. It is this partially filled energy range from $-\omega_{0}$ and $\omega_{0}$ which is responsible for the non-equilibrium properties of the system.

Figure 5. The tunneling processes $s_{0}$ (a) and $s_{t}$ (b). In the process $s_{0}$, both at time 0 and $t$, a quasiparticle tunnels from the left branch to the right branch, and another quasiparticle tunnels in the opposite direction. In the $s_{t}$ process, at time 0 two quasiparticles tunnel from, say, the left to the right branch, and at time $t$ the two quasiparticles tunnel back in the opposite direction. The process $s_{t}$ is responsible for the singularity at the electron frequency $\tilde{\omega}_{0}=2 \omega_{0}$.

Figure 6. Plots for the renormalized noise $\tilde{S}$ vs. $\tilde{\omega} / \tilde{\omega}_{0} . \tilde{S}, \tilde{\omega}$ and $\tilde{\omega}_{0}$ are the renormalized noise and frequencies, using the coupling constant as the scaling factor $\left(\tilde{S}=\frac{S}{2|\Gamma|^{2}}, \tilde{\omega}=\frac{\omega}{4 \pi|\Gamma|^{2}}\right.$ and $\left.\tilde{\omega}_{0}=\frac{\omega_{0}}{4 \pi|\Gamma|^{2}}\right)$. In (a) the excess noise $\tilde{S}-\tilde{S}^{\tilde{\omega}_{0}=0}$ is plotted for large values of $\tilde{\omega}_{0}$, which illustrate the weak coupling $(|\Gamma| \rightarrow 0)$ limit. The rescaled excess noise $\left(\tilde{S}-\tilde{S}^{\tilde{\omega}_{0}=0}\right) / \tilde{\omega}_{0}^{3}$ is plotted in (b). It shows the strong coupling limit $(|\Gamma| \rightarrow \infty)$ as $\omega_{0} \rightarrow 0$. The full noise $\tilde{S} / \tilde{\omega}_{0}$ is plotted in figure (c). For the larger values of $\omega_{0}$, notice that the singularity at $\tilde{\omega}=2 \tilde{\omega}_{0}$ is hidden in the full noise. Meanwhile, some reminiscent signs of the quasiparticle singularity appear near $\tilde{\omega}=\tilde{\omega}_{0}$.

Figure 7. The association of the four densities $\rho_{i}(i=1,2,3,4)$ to the left and right moving branches for the dual pictures corresponding to (a) $g=\nu$ and (b) $g=\nu^{-1}$ (compare to Figs. 1a and 1b). Notice that $\rho_{3}$ and $\rho_{4}$ change chirality under duality, and that the space coordinates (the $x$ and $\tilde{x}$ axis) should also be redefined under the duality transformation.

Figure 8. An insertion of an operator $e^{+i q \phi(t)}$ corresponds to the insertion of a charge + on the contour at time $t$. Similarly, an insertion of an operator $e^{-i q \phi(t)}$ corresponds to an insertion of a charge - at time $t$. The time $t$ is ordered along the contour shown, and there is a distinction between charges placed on the top and bottom branches. In the illustration, we consider the particular case when the - charge is inserted on the top contour, and the + charge is inserted on the bottom contour. 
Fig. 1

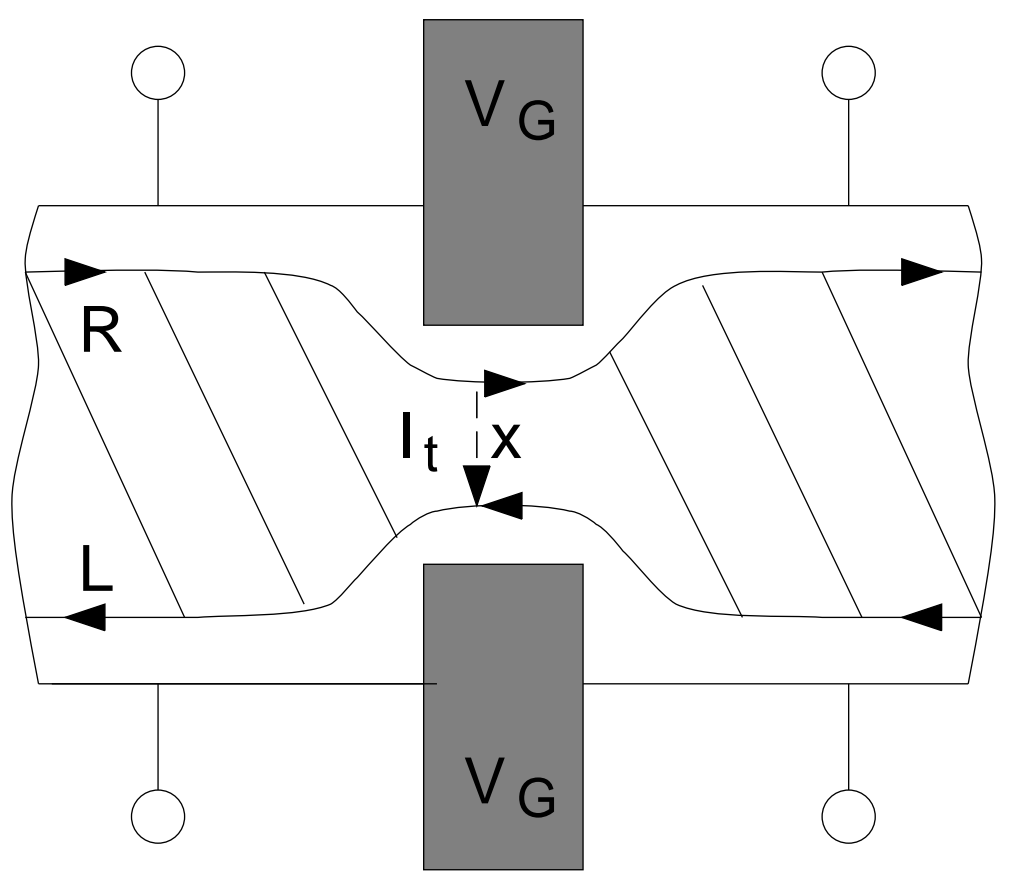

(a)

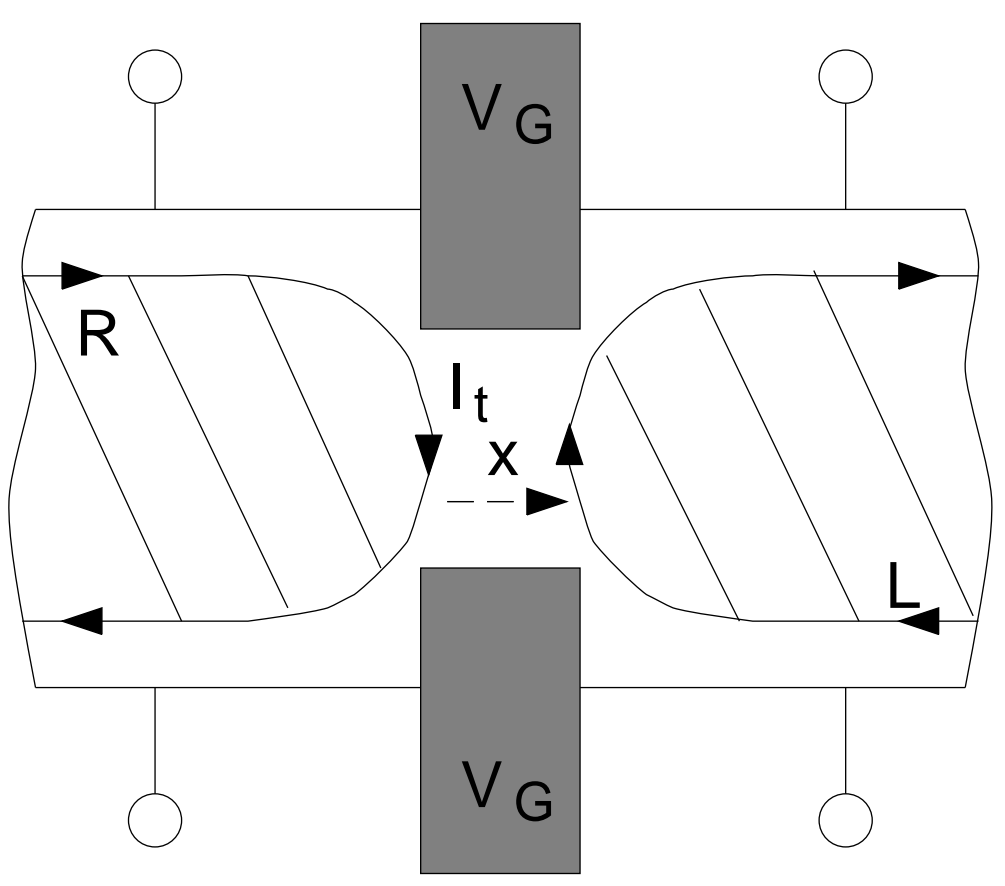

(b) 
Fig. 2

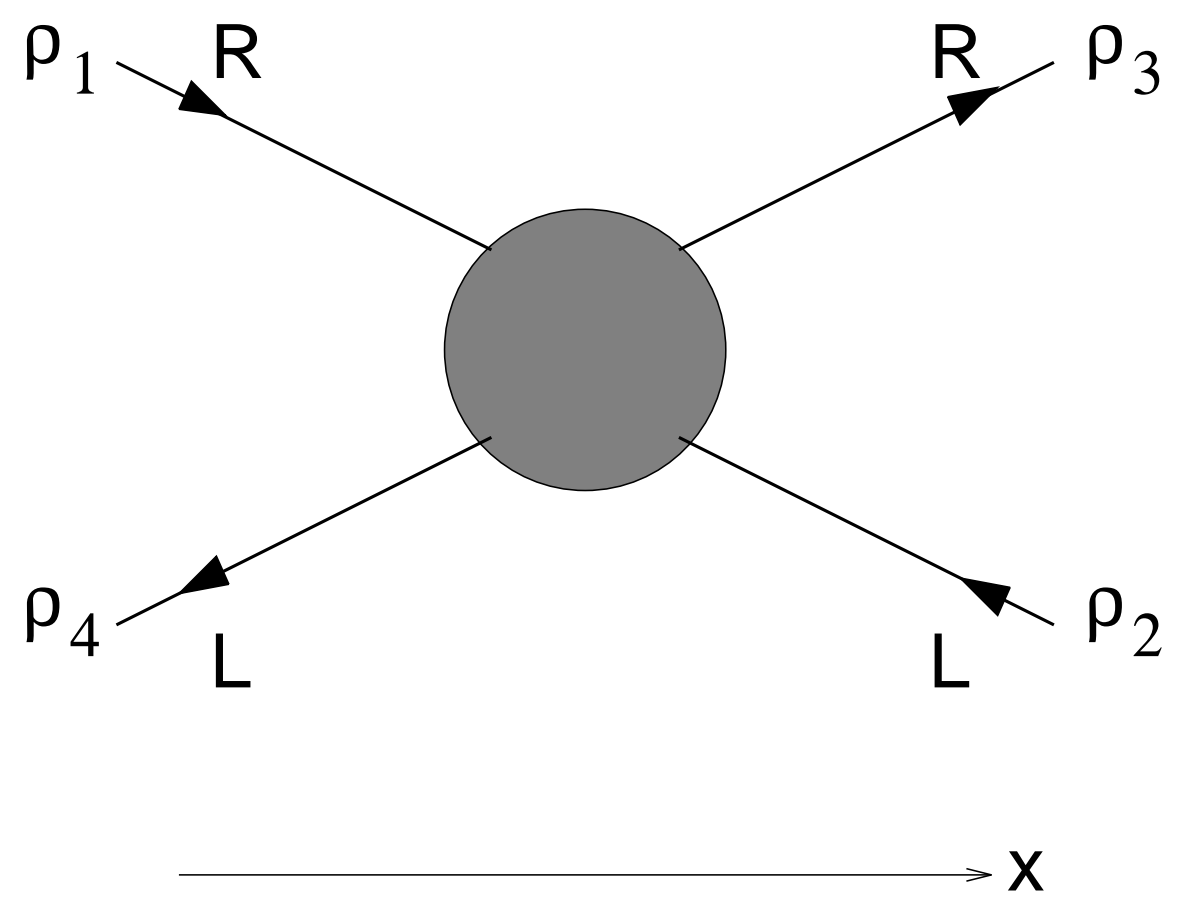


Figure 3

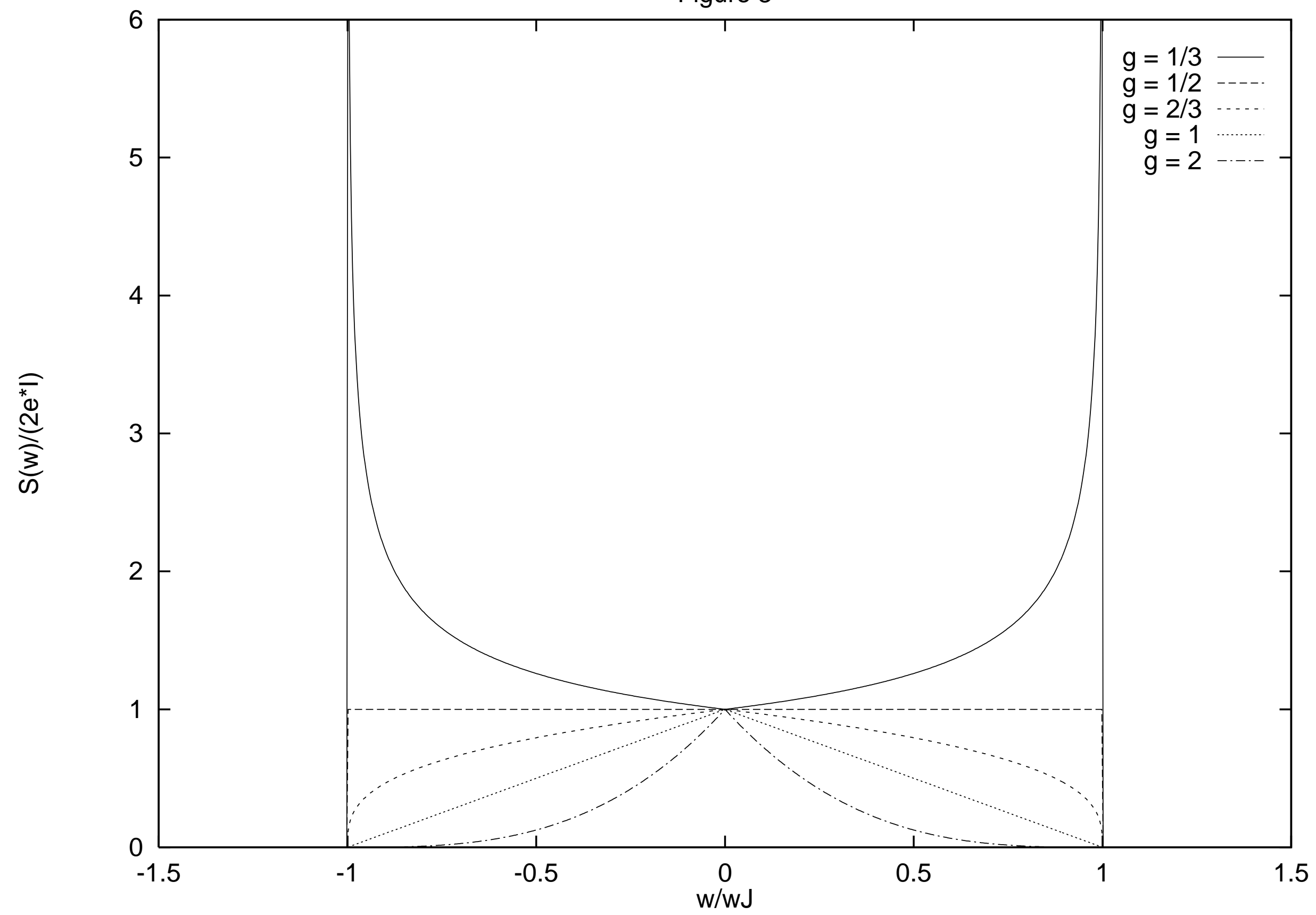


Fig. 4

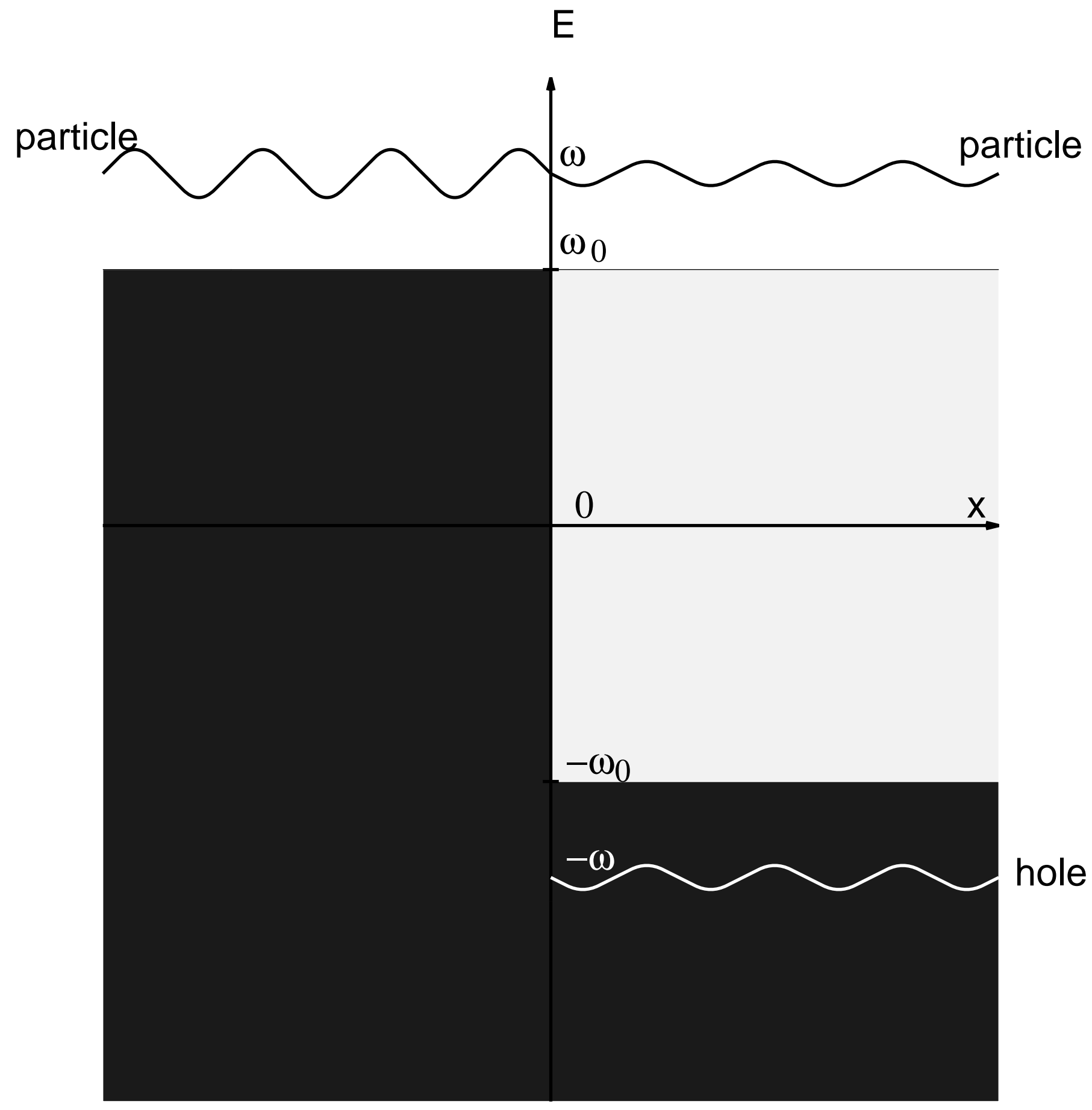


Fig. 5
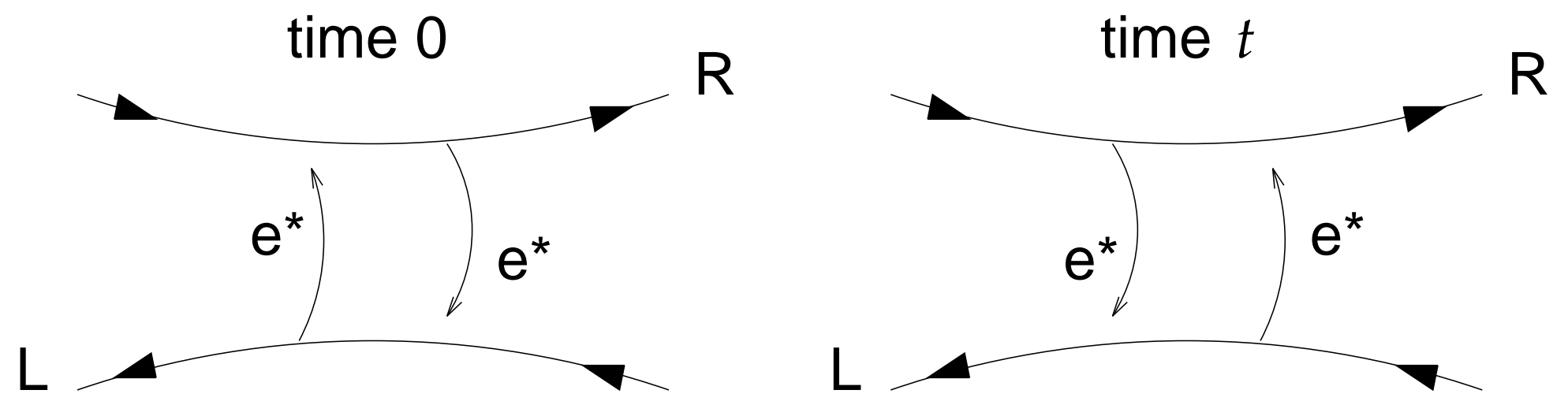

$\mathrm{S}_{\mathrm{O}}$

(a)
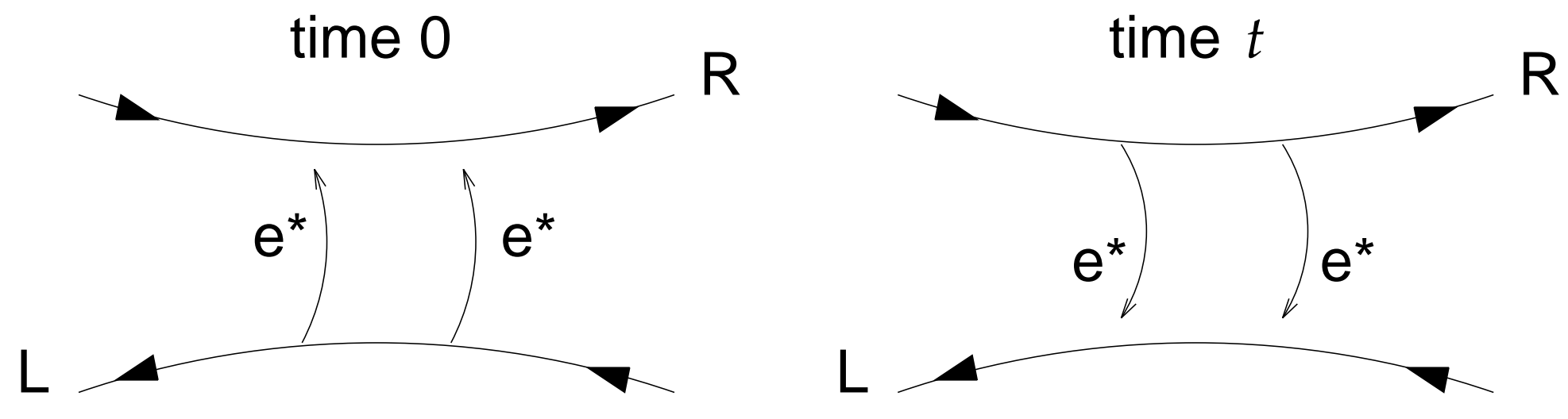

$\mathrm{S}_{\mathrm{T}}$

(b) 
Figure $6 a$

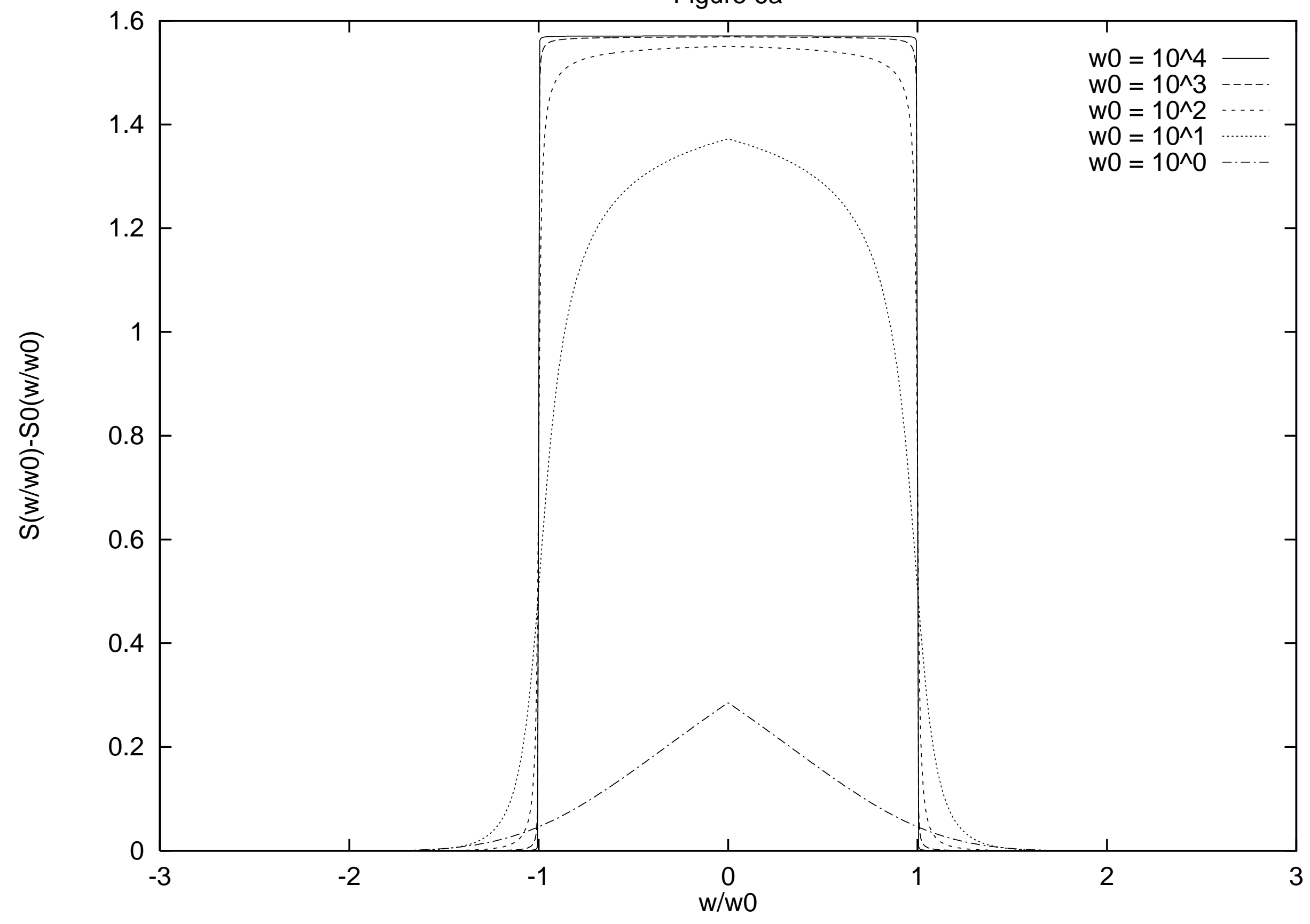


Figure $6 b$

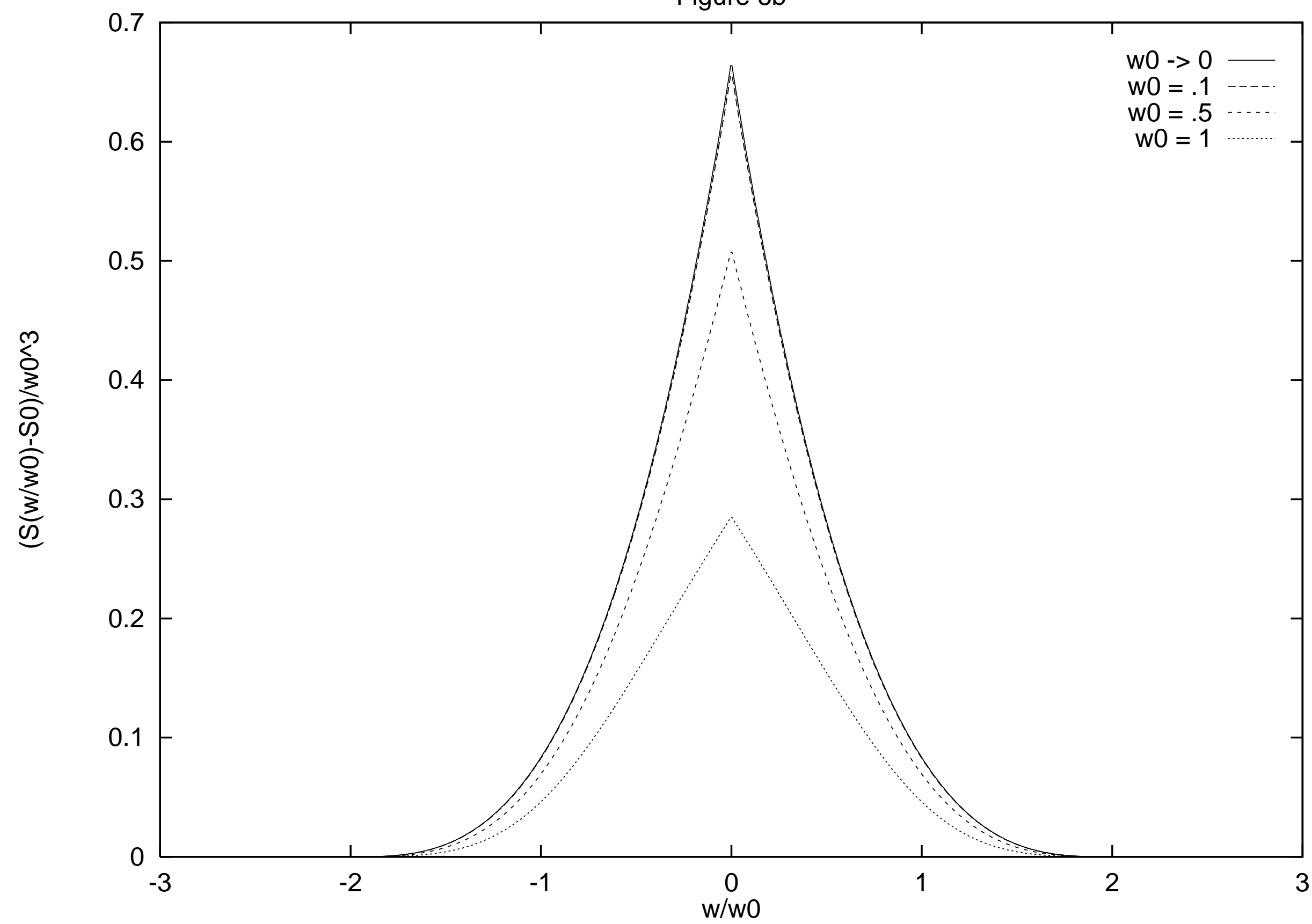


Figure 6c

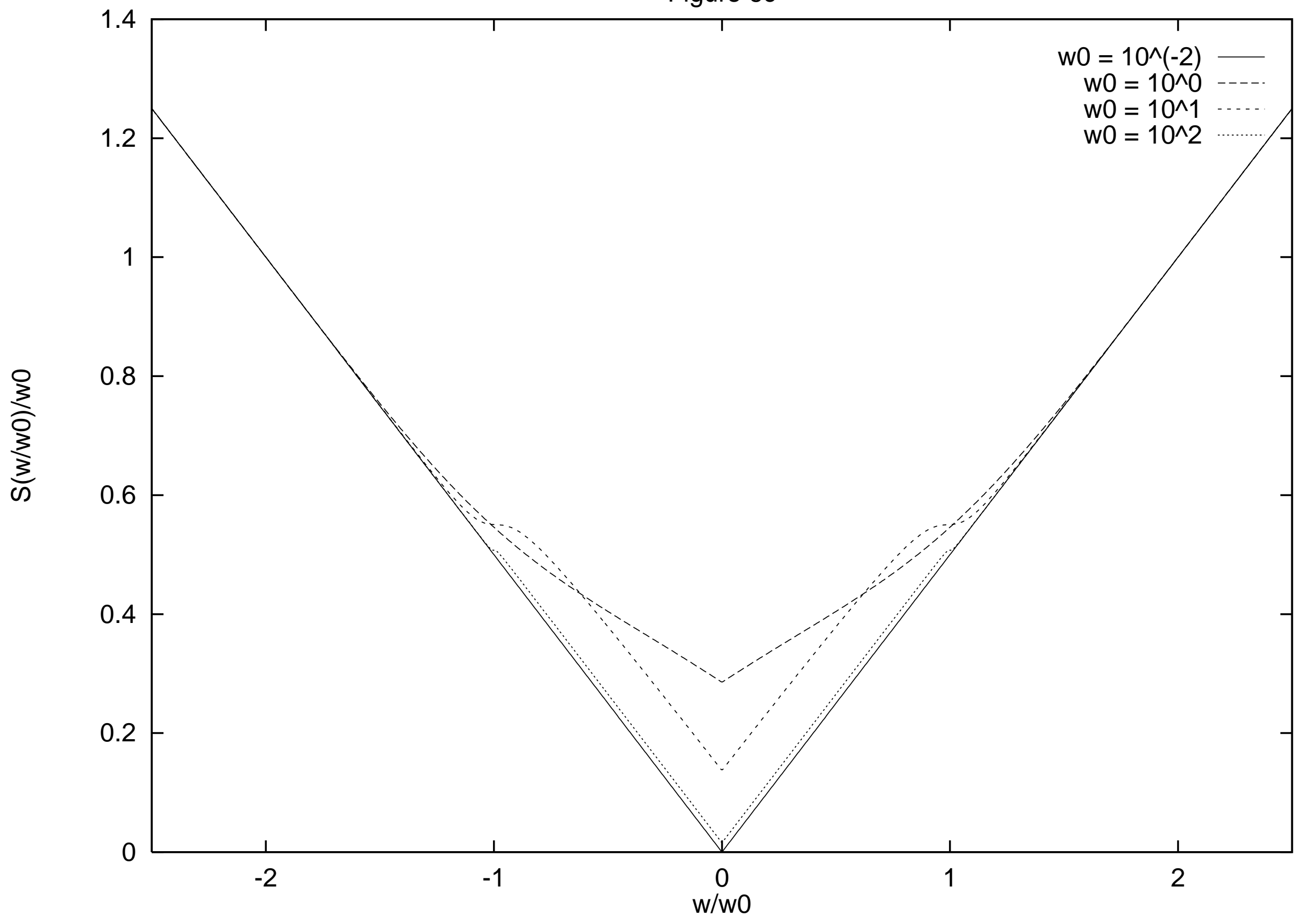


Fig. 7

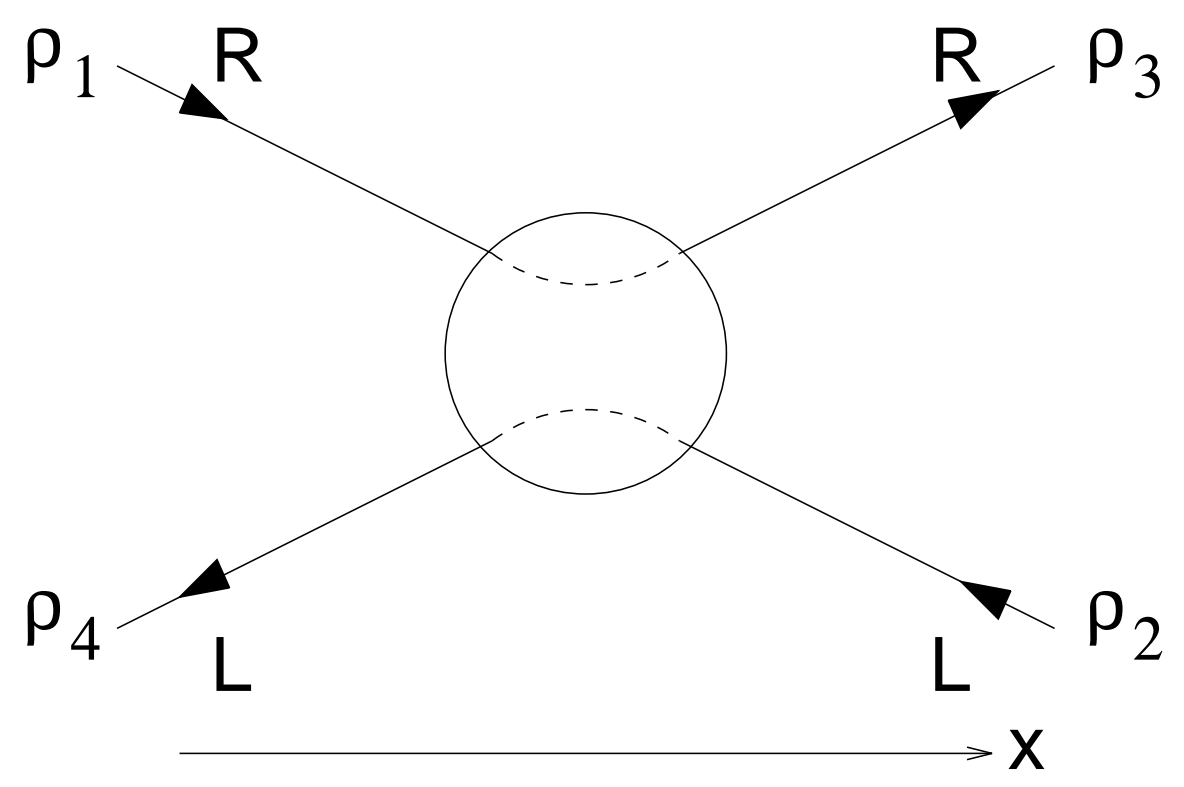

(a)

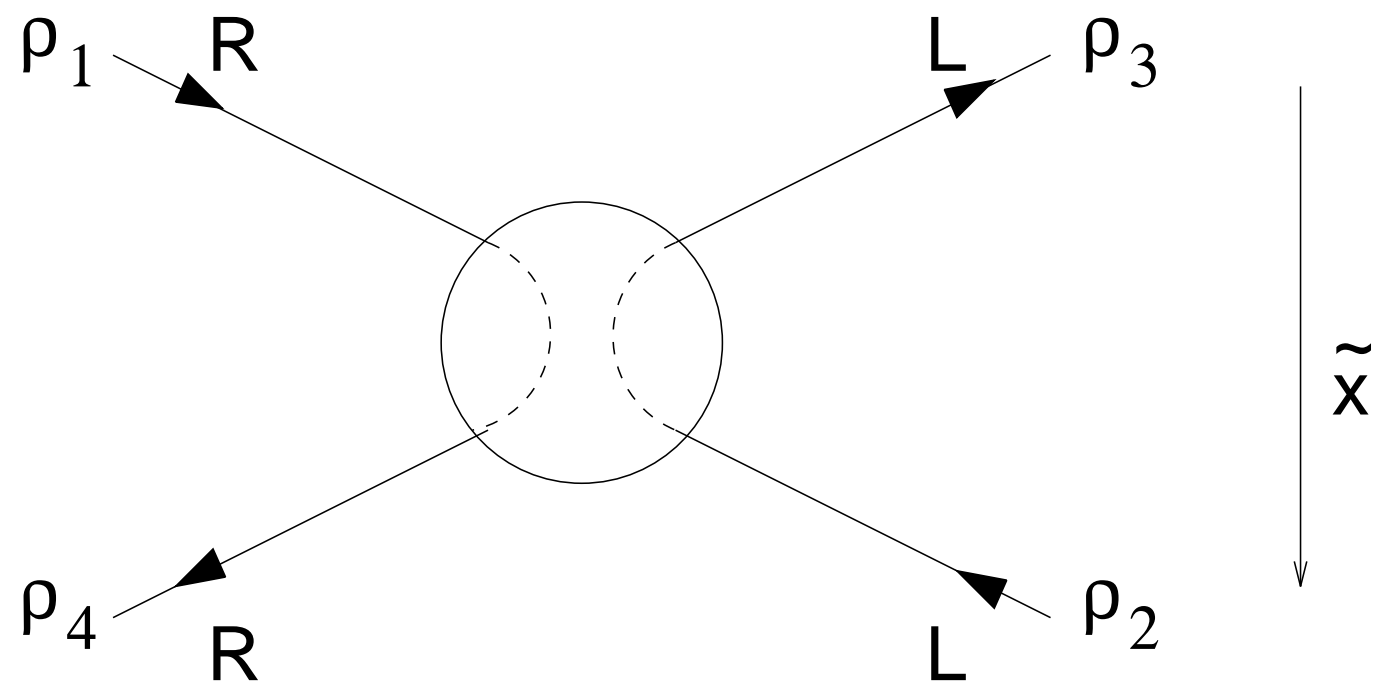

(b) 
Fig. 8
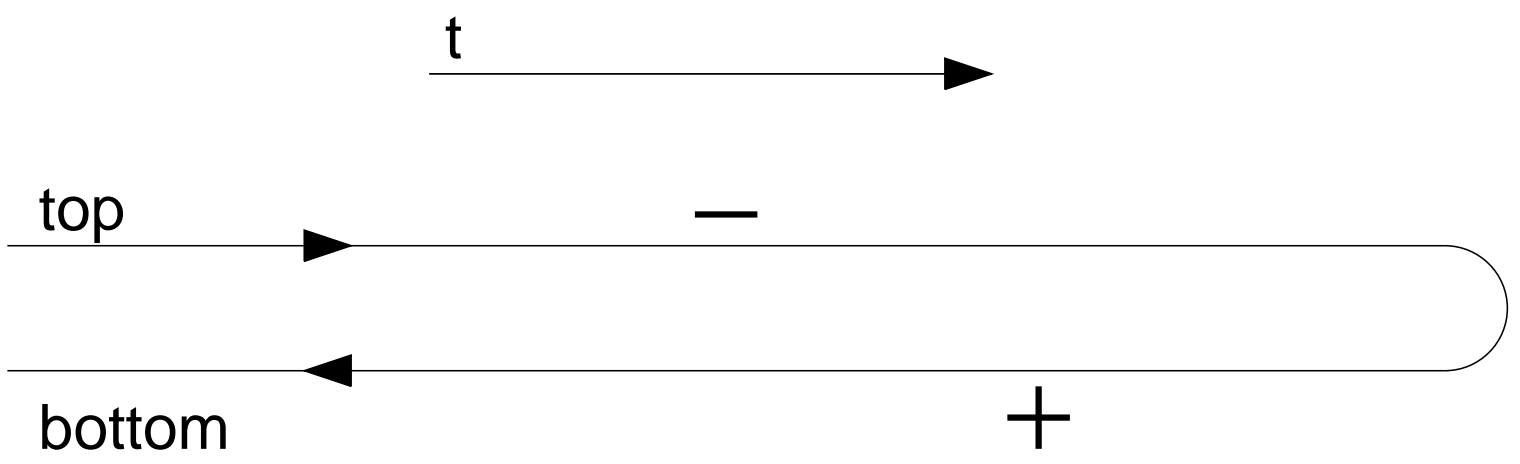